\title{
QUANTITATIVE GRADIENT ESTIMATES FOR HARMONIC MAPS INTO SINGULAR SPACES
}

\author{
HUI-CHUN ZHANG, XIAO ZHONG, AND XI-PING ZHU
}

Dedicated to Professor Yang Lo on the Occasion of his 80th Birthday

\begin{abstract}
In this paper, we will show the Yau's gradient estimate for harmonic maps into a metric space $\left(X, d_{X}\right)$ with curvature bounded above by a constant $\kappa, \kappa \geqslant 0$, in the sense of Alexandrov. As a direct application, it gives some Liouville theorems for such harmonic maps. This extends the works of S. Y. Cheng [4] and H. I. Choi [5] to harmonic maps into singular spaces.
\end{abstract}

\section{Contents}

1. Introduction

1.1. Yau's gradient estimates for harmonic maps into metric spaces

1.2. A sharp Bochner inequality for the harmonic maps into metric spaces

1.3. The outline of the proof of the Bochner inequality

2. Preliminaries

2.1. Energy and Sobolev spaces of maps into metric spaces

2.2. $C A T(\kappa)$-spaces

2.3. Harmonic maps

2.4. Generalized Rademacher theorem for Lipschitz maps

3. An asymptotic mean value inequality

4. The Bochner inequality for harmonic maps into $C A T(\kappa)$-spaces

4.1. Auxiliary functions

4.2. The Bochner inequality

5. Yau's gradient estimates

Appendix A. An generalized Jensen's lemma and the proof of Lemma 4.3.

References

\section{INTRODUCTION}

Let $M, N$ be two smooth Riemannian manifolds. There is a natural concept of energy functional for $C^{1}$-maps between $M$ and $N$. The local minimizers (or more general critical points) of such an energy functional are called harmonic maps. Regularity of harmonic maps is an important topic in the field of geometric analysis. If $\operatorname{dim} M=2$, the regularity of energy minimizing harmonic maps was established by C. Morrey [37]. If $\operatorname{dim} M \geqslant 3$, a beautiful regularity theory was established by Schoen-Uhlenbeck [41, 42], and in a somewhat different context, by Giaquinta-Giusti [16, 17] (and by [22] when the image of the map is contained in a convex ball of $N$ ).

2010 Mathematics Subject Classification. 58E20.

Key words and phrases. Harmonic maps, Bochner formula, CAT $(\kappa)$-spaces, Liouville theorem. 
In 1975, Yau established a seminal interior gradient estimate for harmonic functions on Riemanian manifolds with Ricci curvature bounded below. In 1980, Cheng [4] generalized the Yau's gradient estimate to harmonic maps.

Theorem 1.1. (Cheng [4]) Let $M, N$ be complete Riemannian manifolds such that $M$ has Ricci curvature $\operatorname{Ric}_{M} \geqslant-K, K \geqslant 0$, and that $N$ is simply-connected and is having non-positive sectional curvature. Let $f: M \rightarrow N$ be a harmonic map. Assume that $f\left(B_{a}\left(x_{0}\right)\right) \subset B_{b}\left(y_{0}\right)$ for some $x_{0} \in M, y_{0} \in N$ and some $a, b>0$. Then we have

$$
\sup _{B_{a / 2}\left(x_{0}\right)}|\nabla f|^{2} \leqslant C_{n} \cdot \frac{b^{4}}{a^{4}} \cdot \max \left\{\frac{K a^{4}}{b^{2}}, \frac{a^{2}\left(1+K a^{2}\right)}{b^{2}}, \frac{a^{2}}{b^{2}}\right\},
$$

where $C_{n}$ is a constant depending only on $n=\operatorname{dim}(M)$.

In particular, when $K=0$, this implies a Liouville theorem: if the $f$ is bounded, then it is a constant map. Choi [5] further extended Cheng's work [4] as following.

Theorem 1.2. (Choi [5] ). Let $M, N$ be complete Riemannian manifolds such that $M$ has Ricci curvature $\operatorname{Ric}_{M} \geqslant-K, K \geqslant 0$, and that $N$ has sectional curvature $\sec _{N} \leqslant \kappa, \kappa>0$. Let $f: M \rightarrow N$ be a harmonic map. Assume that $f(M) \subset B_{b}\left(y_{0}\right)$ lies inside the cut locus of $y_{0} \in N$ and some $b<\pi /(2 \sqrt{\kappa})$. Then $|\nabla f|$ is bounded by a constant depending only on $n, K, \kappa$ and $b$. If, furthermore, $K=0$, then $f$ is a constant map.

It is well known from [22, 25] that the radius $b<\pi /(2 \sqrt{\kappa})$ is sharp. Without the restriction that the image of $u$ is contained in a ball with radius $\pi /(2 \sqrt{\kappa})$, a harmonic map might not be even continuous.

\subsection{Yau's gradient estimates for harmonic maps into metric spaces.}

The purpose of this paper is to extend the Yau's gradient estimate to harmonic maps into singular metric spaces.

In the seminal work of M. Gromov and R. Schoen [19], they initiated to study harmonic maps into singular spaces. A general theory of harmonic maps between singular spaces was developed by Korevaar-Schoen [31], Jost [26, 28] and Lin [33], independently.

If $u$ is a map from a domain $\Omega \subset M$ of Riemannian manifold to an arbitrarily metric space $\left(X, d_{X}\right)$, which is unnecessary to be embedded into a Euclidean space, N. Korevaar and R. Schoen [31] introduced an intrinsic approach to generalize the concept of the energy of $u$. Given a map $u \in L^{2}(\Omega, X)$, for each $\epsilon>0$, the approximating energy $E_{\epsilon}^{u}$ is defined as a functional on $C_{0}(\Omega)$ :

$$
E_{\epsilon}^{u}(\phi):=\int_{\Omega} \phi(x) e_{\epsilon}^{u}(x) \mathrm{d} v_{g}(x)
$$

where $\phi \in C_{0}(\Omega)$, the space of continuous functions compactly supported on $\Omega$, and $e_{\epsilon}^{u}$ is approximating energy density defined by

$$
e_{\epsilon}^{u}(x):=\frac{n(n+2)}{\omega_{n-1} \cdot \epsilon^{n}} \int_{B_{\epsilon}(x) \cap \Omega} \frac{d_{X}^{2}(u(x), u(y))}{\epsilon^{2}} \mathrm{~d} v_{g}(y),
$$

where $\omega_{n-1}$ is the volume of $(n-1)$-sphere $\mathbb{S}^{n-1}$ with the standard metric. In [31], KorevaarSchoen proved that

$$
\lim _{\epsilon \rightarrow 0^{+}} E_{\epsilon}^{u}(\phi)=E^{u}(\phi)
$$

for some positive functional $E^{u}(\phi)$ on $C_{0}(\Omega)$. The limit functional $E^{u}$ is called the energy (functional) of $u$. By Riesz representation theorem, the nonnegative functional $E^{u}$ is a Radon measure 
on $\Omega$. Moreover, Korevaar-Schoen in [31] proved that the measure is absolutely continuous respect to the Riemannian volume $\operatorname{vol}_{g}$. Denote $e_{u}:=\frac{d E^{u}}{d \mathrm{vol}_{g}}$, the energy density of $u$. For a smooth map $f$ between two smooth Riemannian manifolds, we have $e_{f}=$ const $\cdot|\nabla f|^{2}$.

The (local) minimizing maps, in the sense of calculus of variations, of such an energy functional $E^{u}$ are called harmonic maps.

If $\left(X, d_{X}\right)$ is a locally compact Riemannian simplicial complex with (globally) non-positive curvature in the sense of Alexandrov, Gromov-Schoen [19] established the local Lipschitz regularity for harmonic maps from $\Omega$ to $X$. Korevaar-Schoen [31] extended to the case where $X$ is a general $C A T(0)$-space, a metric space with non-positive curvature in the sense of Alexandrov. A further extension was given by Serbinowski [43]. Let us put these regularity results together as follows.

Theorem 1.3. (Korevaar-Schoen [31, Theorem 2.4.6], Serbinowski [43, Corollary 2.18]). Let $\Omega \subset M$ be a bounded domain (with smooth boundary) of a Riemannian manifold $(M, g)$ and let $\left(X, d_{X}\right)$ be a CAT $(\kappa)$-space for some $\kappa \geqslant 0$. Suppose that $u: \Omega \rightarrow X$ is a harmonic map. Assume that the image of $u$ is contained in a ball with radius $\rho<\pi /(2 \sqrt{\kappa})$. Here and in the sequel, if $\kappa=0$, we always understand $\pi /(2 \sqrt{\kappa})=+\infty$. Then $u$ is locally Lipschitz continuous in $\Omega$. Moreover, for any ball $B_{R}(o) \subset \subset \Omega$, it holds the following Bernstein-type gradient estimate

$$
\sup _{B_{R / 2}(o)} e_{u} \leqslant C f_{B_{R}(o)} e_{u} \mathrm{~d} v_{g}
$$

where the constant $C$ depends on $n=\operatorname{dim}(M), R$, the injectivity radius of $o, \pi /(2 \sqrt{\kappa})-\rho$, and the $C^{1}$-norm of metric coefficients $g$ on $B_{R}(o)$. Here and in the sequel, $f_{E}:=\frac{1}{\operatorname{vol}_{g}(E)} \int_{E}$ denotes the average integral over the measurable set $E$.

In the last two decades, many regularity results have been obtained for (energy minimizing) harmonic maps into or between singular spaces (see, for example, [31, 27, 28, 45, 3, 11, 14, 7, 9, 33, 34, 47, 20, 2] and so on).

For the case when the domain $\Omega$ has nonnegative sectional curvature and the target $X$ is a $C A T(0)$-simplicial complex, J. Chen [3] showed that the constant $C$ in (1.2) depends only on $n$. When the target $X$ is a general $C A T(0)$-space, Jost [29] gave an approach to deduce an explicit bound of the constant in (1.2) in terms of the sectional curvature of $M, n$ and $R$. Other quantitative Lipschitz estimates of $u$ were also given in [7, 9].

In [29, Sect. 6, Page 167], J. Jost proposed an open problem, in the case when the target $X$ is a $C A T(0)$-space, to ask if the $\sup _{B_{R / 2}(o)} e_{u}$ can be dominated by a constant depending only on the lower bound for the Ricci curvature of $M$, the dimension of $M$, and the energy of $u$. Furthermore, a natural problem was arisen from the combination of the Jost's problem and the Cheng's work [4] to ask if a Yau-type interior gradient estimate holds for harmonic map into a $C A T(0)$-space. The first result in this paper answers this problem affirmatively.

Theorem 1.4. Let $\Omega$ be a bounded domain (with smooth boundary) of an n-dimensional Riemannian manifold $(M, g)$ with iic $_{M} \geqslant-K$ for some $K \geqslant 0$, and let $\left(X, d_{X}\right)$ be a CAT(0)-space. Suppose that $u: \Omega \rightarrow X$ is a harmonic map. Given any ball $B_{R}\left(x_{0}\right)$ with $B_{2 R}\left(x_{0}\right) \subset \subset \Omega$, if $u\left(B_{R}\left(x_{0}\right)\right) \subset B_{\rho}\left(Q_{0}\right)$ for some $Q_{0} \in X$ and some $\rho>0$, then we have

$$
\sup _{B_{R / 2}\left(x_{0}\right)} \operatorname{Lip} u \leqslant C_{n, \sqrt{K} R} \cdot \frac{\rho}{R}
$$


where Lipu is the pointwise Lipschitz constant given by

$$
\operatorname{Lip} u(x):=\limsup _{y \rightarrow x} \frac{d_{X}(u(x), u(y))}{d(x, y)},
$$

and where $d(x, y)$ is the distance with respect to the Riemannian metric $g$ on $M$, and $C_{n, \sqrt{K} R}$ is a constant depending only on $n$ and $\sqrt{K} R$.

Remark 1.5. (1) It is clear from the definitions of $e_{u}$ and Lipu that $e_{u}(x) \leqslant(n+2) \operatorname{Lip}^{2} u(x)$ for almost all $x \in \Omega$.

(2) By the fact $\Delta d_{X}^{2}\left(u(x), u\left(x_{0}\right)\right) \geqslant 2 e_{u} \geqslant 0$ (see [28] or Lemma 2.5], it is well-known that the $\sup _{x \in B_{R / 2}\left(x_{0}\right)} d_{X}^{2}\left(u(x), u\left(x_{0}\right)\right)$ can be dominated by $C_{n, \sqrt{K} R} R^{2} \cdot f_{B_{R}\left(x_{0}\right)} e_{u} d v_{g}$ (see, for example, Eq.(2.7)). So, by choosing $Q_{0}=u\left(x_{0}\right)$, Theorem 1.4 implies that

$$
\sup _{B_{R / 2}\left(x_{0}\right)} \operatorname{Lip} u \leqslant C_{n, \sqrt{K} R}\left(f_{B_{R}\left(x_{0}\right)} e_{u} \mathrm{~d} v_{g}\right)^{1 / 2} .
$$

It answers the Jost's problem ([29, Sect. 6]) affirmatively.

As an immediate application of Theorem 1.4, by letting $R \rightarrow \infty$, we have the following Liouville theorem (cf. [44, Theorem 1.4] and [23, Theorem 1.2]).

Corollary 1.6. Let $(M, g)$ be an n-dimensional complete non-compact Riemannian manifold with nonnegative Ricci curvature, and let $\left(X, d_{X}\right)$ be a CAT(0)-space. Let $u: M \rightarrow X$ be a harmonic map. If u satisfies sub-linear growth:

$$
\liminf _{R \rightarrow \infty} \frac{\sup _{y \in B_{R}\left(x_{0}\right)} d_{X}\left(u(y), Q_{0}\right)}{R}=0
$$

for some $Q_{0} \in X$, then u must be a constant map.

For the case when the target space has curvature $\leqslant \kappa$ for some $\kappa>0$, we have the following gradient estimate.

Theorem 1.7. Let $\Omega$ be a bounded domain (with smooth boundary) of an n-dimensional Riemannian manifold $(M, g)$ with Ric $_{M} \geqslant-K$ for some $K \geqslant 0$, and let $\left(X, d_{X}\right)$ be a CAT $(\kappa)$-space, $\kappa>0$. Suppose that $u: \Omega \rightarrow X$ is a harmonic map with the image $u(\Omega) \subset B_{\rho}\left(Q_{0}\right)$ for some $Q_{0} \in X$ and $\rho<\pi /(2 \sqrt{\kappa})$. Then we have

$$
\sup _{B_{R / 2}\left(x_{0}\right)} \operatorname{Lip} u \leqslant \frac{C_{n, \sqrt{K} R, \pi /(2 \sqrt{\kappa})-\rho}}{R},
$$

where $C_{n, \sqrt{K} R, \pi /(2 \sqrt{\kappa})-\rho}$ is a constant depending only on $n, \sqrt{K} R$ and $\pi /(2 \sqrt{\kappa})-\rho$.

This implies the following Liouville theorem, by letting $R \rightarrow \infty$.

Corollary 1.8. Let $(M, g)$ be an n-dimensional complete non-compact Riemannian manifold with nonnegative Ricci curvature, and let $\left(X, d_{X}\right)$ be a CAT(1)-space. Let $u: M \rightarrow X$ be a harmonic map. If $u(M) \subset B_{\rho}\left(Q_{0}\right)$ for some $Q_{0} \in X$ and $\rho<\pi / 2$, then u must be a constant map.

Remark 1.9. If $u(M) \subset B_{\pi / 2}\left(Q_{0}\right)$ and if $d_{X}^{2}\left(Q_{0}, u(x)\right) \in L^{1}(M)$, then the same conclusion, $u$ is a constant map, has been proved recently by B. Freidin and Y. Zhang in [13]. 


\subsection{A sharp Bochner inequality for the harmonic maps into metric spaces.}

Cheng's argument in [4] is based on the classical Bochner formula of Eells and Sampson. That is, for a smooth harmonic map $u$ between two Riemannian manifolds $M$ and $N$, it holds:

$$
\begin{aligned}
\frac{1}{2}|\nabla u|^{2} & =|d \nabla u|^{2}+\operatorname{Ric}_{M}(\nabla u, \nabla u)-\left\langle R^{N}\left(u_{*} e_{\alpha}, u_{*} e_{\beta}\right) u_{*} e_{\alpha}, u_{*} e_{\beta}\right\rangle \\
& \geqslant\left.|\nabla| \nabla u\right|^{2}-K|\nabla u|^{2}-\kappa|\nabla u|^{4},
\end{aligned}
$$

where the Ricci curvature of $M$ is bounded below by $-K$ and the sectional curvature of $N$ is bounded above by $\kappa$. It is clear that the classical Bochner formula relies heavily on the smoothness of the target space $X$ (requiring to have at least second order derivatives).

For harmonic maps into singular spaces, it is a basic problem to deduce a Bochner type formula. For the case when the domain $\Omega$ has nonnegative sectional curvature and the target $X$ is a non-positively curved simplicial complex, J. Chen [3] used the method in [19] to show that $e_{u}$ is a sub-harmonic function on $\Omega$. In [31], Korevaar-Schoen developed a finite difference technique to prove the following weak form of the Bochner type inequality: there exists a constant $C$, depending on the $C^{1}$-norm of $g$, such that

$$
\int_{\Omega} e_{u}(\Delta \eta+C|\nabla \eta|+C \eta) \mathrm{d} v_{g} \geqslant 0
$$

for all $\eta \in C_{0}^{\infty}(\Omega)$. Korevaar-Schoen's method in [31] has been extended by Serbinowski [43] to the case when the target space is of $C A T(\kappa)$ for any $\kappa>0$. Mese [35] showed that $\Delta e_{u} \geqslant-2 \kappa e_{u}^{2}$, in the sense of distributions, for a harmonic map from a flat domain to a $C A T(\kappa)$-space. Recently, Freidin [12] and Freidin-Zhang [13] improved the method in [19] to deduce the following Bochner type inequality for a harmonic map from a Riemannian manifold into a $C A T(\kappa)$-space:

$$
\frac{1}{2} \Delta e_{u} \geqslant-K e_{u}-\kappa e_{u}^{2}
$$

in the sense of distributions.

Recalling the arguments of Cheng [4] and Choi [5], the key intergradient is the positive term $|\nabla| \nabla u \|^{2}$ in the right hand side of (1.4). The Bochner inequality (1.5) is not enough to get the Theorem 1.4 and Theorem 1.7. In this paper, we will establish a generalized Bochner inequality keeping such a positive term as follows.

Theorem 1.10. Let $\Omega$ be a smooth domain of an n-dimensional Riemannian manifold $(M, g)$ with Ric $_{M} \geqslant-K$ for some $K \geqslant 0$, and let $\left(X, d_{X}\right)$ be a CAT $(\kappa)$-space for some $\kappa \geqslant 0$. Suppose that the map $u: \Omega \rightarrow X$ is harmonic and that its image $u(\Omega)$ is contained in a ball $B_{\rho} \subset X$ with radius $\rho<\frac{\pi}{2 \sqrt{\kappa}}$ if $\kappa>0$.

Then Lipu is in $W_{\mathrm{loc}}^{1,2}(\Omega) \cap L_{\mathrm{loc}}^{\infty}(\Omega)$ and satisfies the following

$$
\frac{1}{2} \Delta \operatorname{Lip}^{2} u \geqslant|\nabla \operatorname{Lip} u|^{2}-K \cdot \operatorname{Lip}^{2} u-\kappa e_{u} \cdot \operatorname{Lip}^{2} u
$$

in the sense of distributions.

\subsection{The outline of the proof of the Bochner inequality.}

In the following, we would like to give a outline of the proof of Theorem 1.10 . First, by the Chain rule, one easily checks that (1.6) is equivalent to

$$
\Delta \operatorname{Lip} u \geqslant-K \cdot \operatorname{Lip} u-\kappa e_{u} \cdot \operatorname{Lip} u
$$

in the sense of distributions. We will first to show that, for any $q \in(1,2]$

$$
\Delta\left(\operatorname{Lip}^{q} u / q\right) \geqslant-K \cdot \operatorname{Lip}^{q} u-\kappa e_{u} \cdot \operatorname{Lip}^{q} u
$$


in the sense of distributions, and then we check the limit as $q \rightarrow 1$ to get (1.7).

The proof of $(1.7 q)$ is inspired by the classical Hamilton-Jocabi flow. Recall that the classical Hamilton-Jacobi equation, given a function $f$ :

$$
\frac{\partial v(x, t)}{\partial t}=-|\nabla v(x, t)|^{2}
$$

with $v(x, 0)=f(x)$, has a solution (by Hopf-Lax formula)

$$
\left.\mathscr{H}_{t} f(x):=\inf _{y \in B_{R}}\left\{\frac{d^{2}(x, y)}{2 t}+f(y)\right)\right\}, \quad t>0 .
$$

The difference of "time $t$ " to the Hamilton-Jacobi flow $\mathscr{H}_{t} f(x)$ at $t=0$ gives the gradient $-|\nabla f(x)|^{2}$. That is, as $t \rightarrow 0^{+}$,

$$
\frac{\mathscr{H}_{t} f(x)-f(x)}{t} \rightarrow-|\nabla f(x)|^{2} .
$$

This suggests to study the Hamilton-Jacobi flow $\mathscr{H}_{t} f(x)$ for the gradient estimates of $f$.

In order to obtain $(1.7 q)$, we introduce a family of functions $\left(f_{t}\right)_{t>0}$ by: on a fixed ball $B_{R}:=$ $B_{R}(o)$ with $B_{2 R} \subset \subset \Omega$, for any $q \in(1,2]$,

$$
f_{t}(x):=\inf _{y \in B_{2 R}}\left\{\frac{d^{p}(x, y)}{p t^{p-1}}-\phi\left(d_{X}(u(x), u(y))\right)\right\}, \quad \forall x \in B_{R}, \quad \forall t>0,
$$

where $p=q /(q-1)$ and $\phi:[0,1 / 10] \rightarrow \mathbb{R}$ is a suitable smooth convex function with $\phi(0)=0$ and $\phi^{\prime}(0)=1$.

It is easy to check that, for any $x \in B_{R}$ and any sufficiently small $t$, the "inf" of (1.8) can be realized by some point $y_{t, x} \in B_{2 R}$. The set of all such points is denoted by $S_{t}(x)$. Then we put

$$
L_{t}(x):=\min _{y_{t, x} \in S_{t}(x)} d\left(x, y_{t, x}\right) \quad \text { and } \quad D_{t}(x):=\frac{L_{t}^{p}(x)}{p t^{p-1}}-f_{t}(x) .
$$

The proof of (1.7q) contains two parts. Without loss of generality, we may assume $\kappa=1$. Firstly, we shall show that, for any given $\varepsilon>0, f_{t}$ satisfies an elliptic inequality

$$
\Delta f_{t}(x) \leqslant \frac{K}{t^{p-1}} \cdot L_{t}^{p}(x)+(1+\varepsilon) \cdot e_{u}(x) D_{t}(x),
$$

on $B_{R}$, for any sufficiently small $t>0$, in the sense of distributions. Secondly, we want to show that, for almost all $x \in B_{R}$,

$$
\lim _{t \rightarrow 0} \frac{f_{t}}{t}=-\frac{1}{q} \operatorname{Lip}^{q} u, \quad \lim _{t \rightarrow 0^{+}} \frac{L_{t}}{t}=\operatorname{Lip}^{q / p} u, \quad \lim _{t \rightarrow 0^{+}} \frac{D_{t}}{t}=\operatorname{Lip}^{q} u .
$$

The combination of (1.10) and (1.11) will yield the inequality $(1.7 q)$.

In order to prove [1.11), we recall a generalized Rademacher theorem in [30]. Let $f: \Omega \rightarrow X$ be a Lipschitz map, Kirchheim [30] proved for almost all $x \in \Omega$, that there exists a semi-norm, denoted by $m d f_{x}$ and called metric differential, such that

$$
d_{X}\left(f\left(\exp _{x}(t \xi)\right), f(x)\right)-t \cdot m d f_{x}(\xi)=o(t),
$$

for all $\xi \in \mathbb{S}^{n-1} \subset T_{x} M$. By using this result, one can deduce a representative of point-wise Lipschitz constant of $f$ : for almost all $x \in \Omega$,

$$
\operatorname{Lip} f(x):=\max _{\xi \in \mathbb{S}^{n-1}} m d f_{x}(\xi) .
$$

This suffices to show (1.11). See Lemma2.9 and Lemma 4.4 for the details. 
Now, we explain the proof of 1.10], which is inspired by the recent work [47] of the first and the third authors. For simplicity, we assume $\operatorname{Ric}_{M} \geqslant 0$. We need to show that

$$
\Delta f_{t}(x) \leqslant(1+\varepsilon) e_{u}(x) D_{t}(x)+\theta
$$

for sufficiently small $t>0$ and any $\theta>0$ in the sense of distributions. It is a local property, then we need only to consider the case when $R$ is small. We argue by contradictions. Suppose that it fails, by the maximum principle, we have that there exists a domain $U$ and a positive number $\theta_{0}$ such that $f_{t}-v$ achieves a strict minimum in $U$, where $v$ is the solution of Dirichlet problem

$$
\Delta v=(1+\varepsilon) e_{u}(x) D_{t}(x)+\theta_{0} \text { in } U, \quad v=f_{t} \text { on } \partial U .
$$

From the construction of $f_{t}$, we know that the function

$$
H(x, y):=\frac{d^{p}(x, y)}{p t^{p-1}}-F(x, y)-v(x) .
$$

has a minimum in $U \times B_{R}$, where $F(x, y):=\phi\left(d_{X}(u(x), u(y))\right)$. We denote one of these minimum points by $(\bar{x}, \bar{y})$.

Let $T: T_{\bar{x}} M \rightarrow T_{\bar{y}} M$ be the parallel transportation from $\bar{x}$ to $\bar{y}$. We want to consider the asymptotic behavior of the average

$$
f_{B_{\epsilon}(0)} H\left(\exp _{\bar{x}}(\eta), \exp _{\bar{y}}(T \eta)\right) \mathrm{d} \eta
$$

as $\epsilon \rightarrow 0$. According to $R i c_{M} \geqslant 0$, by integrating the second variation of arc-length over $B_{\epsilon}(0)$, we have that

$$
f_{B_{\epsilon}(0)}\left(d^{p}\left(\exp _{\bar{x}}(\eta), \exp _{\bar{y}}(T \eta)\right)-d^{p}(\bar{x}, \bar{y})\right) \mathrm{d} \eta \leqslant o\left(\epsilon^{2}\right) .
$$

Notice that $\Delta v=(1+\varepsilon) e_{u}(x) D_{t}(x)+\theta_{0}$ implies that $v$ is smooth near $\bar{x}$, it follows that

$$
-f_{B_{\epsilon}(0)}\left(v\left(\exp _{\bar{x}}(\eta)\right)-v(\bar{x})\right) \mathrm{d} \eta \leqslant-\frac{1}{2(n+2)}\left[(1+\varepsilon) e_{u}(\bar{x}) D_{t}(\bar{x})+\theta_{0}\right] \cdot \epsilon^{2}+o\left(\epsilon^{2}\right) .
$$

Thus, we only need to show an asymptotic mean value inequality that

$$
-f_{B_{\epsilon}(0)}\left(F\left(\exp _{\bar{x}}(\eta), \exp _{\bar{y}}(T \eta)\right)-F(\bar{x}, \bar{y})\right) \mathrm{d} \eta \leqslant \frac{1+\varepsilon}{2(n+2)} e_{u}(\bar{x}) D_{t}(\bar{x}) \cdot \epsilon^{2}+o\left(\epsilon^{2}\right) .
$$

Indeed, once one has proved (1.14), the combination of (1.12)-(1.14) contradicts with the fact that $H(x, y)$ has a minimum at $(\bar{x}, \bar{y})$, and hence it follows (1.10).

In order to show (1.14), we need to choose a suitable function $\phi(s)$ in (1.8). In the simplest case that $\kappa=0$ and $p=q=2$, we can choose directly $\phi(s)=s$, as in [47].

In the case of $\kappa=1$ (and general $q \in(1,2]$ ), the definition of $C A T(1)$ suggests us to choose $\phi(s)=2 \sin (s / 2)$. However, this is not convex for small $s>0$. An exact relation in CAT(1)spaces, Lemma 2.3, suggests us to perturb $2 \sin (s / 2)$ to

$$
\phi(s)=2 \sin (s / 2)+4 \sin ^{2}(s / 2) .
$$

Fortunately, this is convex for small $s>0$.

Given any $a, b \in \mathbb{R}$ with $a, b \geqslant 0$, and fixed any $q \in \Omega, Q \in X$, we define a function near $q$ by

$$
w_{a, b, Q, q}(x):=a \cdot d_{X}^{2}(u(x), u(q))+b \cdot \cos \left(d_{X}(u(x), Q)\right) .
$$

Since $\left(X, d_{X}\right)$ has curvature $\leqslant 1$, by combining that $e_{\epsilon}^{u}$ converge to $e_{u}$ as $\epsilon \rightarrow 0$ and the fact

$$
\Delta \cos \left(d_{X}(u(x), Q)\right) \leqslant-\cos \left(d_{X}(u(x), Q)\right) \cdot e_{u}(x),
$$


we will be able to deduce that, for almost all $q$, an asymptotic mean value inequality for $w_{a, b, Q, q}$ holds (for some subsequence $\epsilon_{j} \rightarrow 0$, see Lemma 3.3 for the explicit statements).

On the other hand, the assumption $\left(X, d_{X}\right)$ having curvature $\leqslant 1$ implies also that, for any $q_{1}, q_{2}$, the function $w_{a_{2}, b, Q_{m}, q_{1}}+w_{a_{1}, b, Q_{m}, q_{2}}$ touches $-F(\cdot, \cdot)$ by above at $\left(q_{1}, q_{2}\right)$ for some suitable constants $a_{1}, a_{2}, b \geqslant 0$, where $Q_{m}$ is the mid-point of $u\left(q_{1}\right)$ and $u\left(q_{2}\right)$ (the details is given in Lemma 2.3). Therefore, we conclude that an asymptotic mean value inequality for $-F(\cdot, \cdot)$ at almost all $\left(q_{1}, q_{2}\right)$ holds (see Eq. (4.12) and Lemma 3.3 for the explicit formulas). First, let us assume briefly that the mentioned asymptotic mean value inequality for $-F(\cdot, \cdot)$ at $(\bar{x}, \bar{y})$. Then we conclude (1.14) in this case. The primary issue is that there is no reason we can assume that the asymptotic mean value inequality for $-F(\cdot, \cdot)$ at $(\bar{x}, \bar{y})$. In this case, we will perturb the function $H(x, y)$ to $H_{1}(x, y):=H(x, y)+\gamma_{\delta}(x, y)$ by a smooth function $\gamma_{\delta}(x, y)$, which is arbitrarily small up to two order derivatives, such that the mentioned asymptotic mean value inequality for $-F(\cdot, \cdot)$ holds at one of minimum of $H_{1}(x, y)$. This argument of perturbation can be ensured by a generalized Jensen's Lemma in the theory of viscosity solutions of second order partial differential equations.

Acknowledgements. The first and third authors are partially supported by NSFC 11521101, The first author is also partially supported by NSFC 11571374 and by "National Program for support of Top-notch Young Professionals". The second author is supported by the Academy of Finland. Part of the work was done when the first author visited the Department of Mathematics and Statistics, University of Jyväskylä for one month in 2016. He would like to thank the department for the hospitality.

\section{Preliminaries}

\subsection{Energy and Sobolev spaces of maps into metric spaces.}

Let $\Omega$ be a bounded open domain of an $n$-dimensional smooth Riemannian manifold $(M, g)$, and let $\left(X, d_{X}\right)$ be a complete metric space. We will write

$$
|x y|:=d(x, y), \quad \forall x, y \in M .
$$

Several equivalent notions of Sobolev space for maps into metric spaces have introduced in [31, 28, 21, 32, 38]. Fix any $p \in[1, \infty)$. A Borel measurable map $u: \Omega \rightarrow X$ is said to be in the space $L^{p}(\Omega, X)$ if it has separable range and, for some (hence, for all) $P \in X$,

$$
\int_{\Omega} d_{X}^{p}(u(x), P) \mathrm{d} v_{g}(x)<\infty .
$$

We equip with a distance in $L^{p}(\Omega, X)$ by

$$
d_{L^{p}}^{p}(u, v):=\int_{\Omega} d_{X}^{p}(u(x), v(x)) \mathrm{d} v_{g}(x), \quad \forall u, v \in L^{p}(\Omega, X) .
$$

Denote by $C_{0}(\Omega)$ the set of continuous functions compactly supported on $\Omega$. Given $p \in[1, \infty)$ and a map $u \in L^{p}(\Omega, X)$, for each $\epsilon>0$, the approximating energy $E_{p, \epsilon}^{u}$ is defined as a functional on $C_{0}(\Omega)$ :

$$
E_{p, \epsilon}^{u}(\phi):=\int_{\Omega} \phi(x) e_{p, \epsilon}^{u}(x) \mathrm{d} v_{g}(x), \quad \forall \phi \in C_{0}(\Omega),
$$

where the approximating energy density is defined by

$$
e_{p, \epsilon}^{u}(x)=e_{p, \epsilon, g}^{u}(x):=\frac{n+p}{c_{n, p} \cdot \epsilon^{n}} \int_{B_{\epsilon}(x) \cap \Omega} \frac{d_{X}^{p}(u(x), u(y))}{\epsilon^{p}} \mathrm{~d} v_{g}(y),
$$


and the constant $c_{n, p}=\int_{\mathbb{S}^{n-1}}\left|x^{1}\right|^{p} \sigma(d x)$, and $\sigma$ is the canonical Riemannian volume on $\mathbb{S}^{n-1}$. In particular, $c_{n, 2}=\omega_{n-1} / n$, where $\omega_{n-1}$ is the volume of $(n-1)$-sphere $\mathbb{S}^{n-1}$ with standard metric. Next, a map $u \in L^{p}(\Omega, X)$ is said to be in $W^{1, p}(\Omega, X)$ if the energy $E_{p}^{u}<\infty$, where

$$
E_{p}^{u}:=\sup _{\phi \in C_{0}(\Omega), 0 \leqslant \phi \leqslant 1}\left(\limsup _{\epsilon \rightarrow 0} E_{p, \epsilon}^{u}(\phi)\right) .
$$

If $1<p<\infty$ and $u \in W^{1, p}(\Omega, X)$, it was proved in [31] that, for each $\phi \in C_{0}(\Omega)$, the limit

$$
E_{p}^{u}(\phi):=\lim _{\epsilon \rightarrow 0^{+}} E_{p, \epsilon}^{u}(\phi)
$$

exists (called $p$-th energy functional of $u$ ), and that $E_{p}^{u}$ is absolutely continuous with respect to the Riemannian volume vol. Denote the density by $e_{u, p} \in L_{\text {loc }}^{1}(\Omega)$. Moreover, from [31, Lemma 1.4.2], there exists a constant $C>0$, independent of $\epsilon$ such that

$$
E_{p, \epsilon}^{u}(\phi) \leqslant E_{p}^{u}\left(C \epsilon \phi+\max _{y \in B_{\epsilon}(x)}|\phi(y)-\phi(x)|\right)
$$

for any sufficiently small $\epsilon>0$. Thus, by Dunfold-Pettis Theorem, it implies that

$$
e_{p, \epsilon}^{u} \rightarrow e_{u, p} \text { in } L_{\mathrm{loc}}^{1}(\Omega), \quad \text { as } \epsilon \rightarrow 0 .
$$

For the special case $p=2$, we write $e_{u}:=e_{u, 2}$ and $E^{u}:=E_{2}^{u}$ for any $u \in W^{1,2}(\Omega, X)$. We summarize some main properties of $W^{1,2}(\Omega, X)$, which can be found in [31, 32].

Proposition 2.1. Let $u \in W^{1,2}(\Omega, X)$.

(1) (Lower semi-continuity) For any sequence $u_{j} \rightarrow u$ in $L^{2}(\Omega, X)$ as $j \rightarrow \infty$, we have

$$
E^{u}(\phi) \leqslant \liminf _{j \rightarrow \infty} E^{u_{j}}(\phi), \quad \forall 0 \leqslant \phi \in C_{0}(\Omega) .
$$

(2) (Equivalence for $X=\mathbb{R}$ ) If $X=\mathbb{R}$, the above space $W^{1,2}(\Omega, \mathbb{R})$ is equivalent to the usual Sobolev space $W^{1,2}(\Omega)$.

(3) (Weak Poincaré inequality, see for example [32, Theorem 4.2]) For any ball $B_{R}(q)$ with $B_{6 R}(q) \subset \subset \Omega$, there exists a constant $C_{n, K, R}>0$ such that the following holds: for any $z \in B_{R}(q)$ and any $r \in(0, R / 2)$, we have

$$
\int_{B_{r}(z)} \int_{B_{r}(z)} d_{X}^{2}(u(x), u(y)) \mathrm{d} v_{g}(x) \mathrm{d} v_{g}(y) \leqslant C_{n, K, R} \cdot r^{n+2} \cdot \int_{B_{6 r}(z)} e_{u}(x) \mathrm{d} v_{g}(x) .
$$

Remark 2.1. By a rescaling argument, one can easily improve the constant $C_{n, K, R}$ in (2.1) to a constant $C_{n, K R^{2}}$ depending only on $n$ and $K R^{2}$. Indeed, let us consider the rescaling the metric on $M$ by $g_{R}:=R^{-2} g$. Then we have $R i c_{g_{R}} \geqslant-K R^{2}$ and $d v_{g_{R}}=R^{-n} d v_{g}$. By the definition of $e_{p, \epsilon, g}^{u}$, we get $e_{p, R^{-1} \epsilon, g_{R}}^{u}=R^{p} \cdot e_{p, \epsilon, g}^{u}$. Therefore, by the definition of $e_{u, p}$, the Poincaré constant in (2.1) is invariant with respect to the rescaling $g \mapsto g_{R}$.

\section{2. $C A T(\kappa)$-spaces.}

Let us review firstly the concept of spaces with curvature bounded above (globally) in the sense of Alexandrov.

Definition 2.2 (see, for example, [1, 11]). A geodesic space $\left(X, d_{X}\right)$ is called to be globally curvature bounded above by $\kappa$ in the sense of Alexandrov, for some $\kappa \in \mathbb{R}$, denoted by $C A T(\kappa)$, if the following comparison property is to hold: Given any triangle $\triangle P Q R \subset X$ such that $d_{X}(P, Q)+$ $d_{X}(Q, R)+d_{X}(R, P)<2 \pi / \sqrt{\kappa}$ if $\kappa>0$ and point $S \in Q R$ with

$$
d_{X}(Q, S)=d_{X}(R, S)=\frac{1}{2} d_{X}(Q, R),
$$


then there exists a comparison triangle $\triangle \bar{P} \bar{Q} \bar{R}$ in the simply connected 2-dimensional space form $\mathbb{S}_{\kappa}^{2}$ with standard metric with sectional curvature $=\kappa$ and point $\bar{S} \in \bar{Q} \bar{R}$ with

$$
d_{\mathbb{S}_{\kappa}^{2}}(\bar{Q}, \bar{S})=d_{\mathbb{S}_{\kappa}^{2}}(\bar{R}, \bar{S})=\frac{1}{2} d_{\mathbb{S}_{\kappa}^{2}}(\bar{Q}, \bar{R})
$$

such that

$$
d_{X}(P, S) \leqslant d_{\mathbb{S}_{K}}(\bar{P}, \bar{S})
$$

It is obvious that $\left(X, d_{X}\right)$ is a $C A T(\kappa)$-space if and only if the rescaled space $\left(X, \sqrt{\kappa} \cdot d_{X}\right)$ is a $C A T(1)$-space, for any $\kappa>0$.

We need a lemma, which follows from [31, Corollary 2.1.3]:

Lemma 2.3. Let $\left(X, d_{X}\right)$ be an $C A T(1)$ space. Take any ordered sequence $\{P, Q, R, S\} \subset X$, and let point $Q_{m}$ be the mid-point of $Q R$. we denote the distance $d_{X}(A, B)$ abbreviatedly by $d_{A B}$. Then, for any $0 \leqslant \alpha \leqslant 1$ and $\beta>0$, we have

$$
\begin{gathered}
\frac{1-\alpha}{2}\left(\left(2 \sin \frac{d_{Q R}}{2}\right)^{2}-\left(2 \sin \frac{d_{P S}}{2}\right)^{2}\right)+\alpha\left(2 \sin \frac{d_{Q R}}{2}\right)\left(2 \sin \frac{d_{Q R}}{2}-2 \sin \frac{d_{P S}}{2}\right) \\
\leqslant\left[1-\frac{1-\alpha}{2}\left(1-\frac{1}{\beta}\right)\right]\left(2 \sin \frac{d_{P Q}}{2}\right)^{2}+2 \cos \frac{d_{Q R}}{2}\left(\cos d_{P Q_{m}}-\cos d_{Q Q_{m}}\right) \\
+\left[1-\frac{1-\alpha}{2}(1-\beta)\right]\left(2 \sin \frac{d_{R S}}{2}\right)^{2}+2 \cos \frac{d_{Q R}}{2}\left(\cos d_{S Q_{m}}-\cos d_{R Q_{m}}\right) .
\end{gathered}
$$

Proof. Consider the embedding $X$ into the cone $C(X)$ with the cone metric $|\cdot \cdot|_{C}$. Then $C(X)$ has non-positive curvature in the sense of Alexandrov. Denote that

$$
\bar{P}=(P, 1), \quad \bar{Q}=(Q, 1), \quad \bar{S}=(S, 1), \quad \bar{R}=(R, 1) \quad \text { and } \quad \bar{Q}_{m}=\left(Q_{m}, 1\right) .
$$

It is clear that the midpoint of $\bar{Q}, \bar{R}$ in $C(X)$ is

$$
\bar{T}=\left(Q_{m}, \cos \frac{d_{Q R}}{2}\right) .
$$

From the equation (2.1v) in [31, Corollary 2.1.3] (by taking $t=1 / 2$ there), we get, for each $\alpha \in[0,1]$, that

$$
\begin{aligned}
|\bar{T} \bar{P}|_{C}^{2}+|\bar{T} \bar{S}|_{C}^{2} \leqslant|\bar{P} \bar{Q}|_{C}^{2}+|\bar{R} \bar{S}|_{C}^{2}+\frac{1}{2}\left(|\bar{S} \bar{P}|_{C}^{2}-|\bar{Q} \bar{R}|_{C}^{2}\right)+\frac{1}{2}|\bar{Q} \bar{R}|_{C}^{2} \\
-\frac{1}{2}\left(\alpha\left(|\bar{S} \bar{P}|_{C}-|\bar{Q} \bar{R}|_{C}\right)^{2}+(1-\alpha)\left(|\bar{R} \bar{S}|_{C}-|\bar{P} \bar{Q}|_{C}\right)^{2}\right)
\end{aligned}
$$

Notice that $|\bar{Q} \bar{R}|_{C}=2|\bar{T} \bar{Q}|_{C}=2|\bar{T} \bar{R}|_{C}$ and that

$$
\left(|\bar{S} \bar{P}|_{C}^{2}-|\bar{Q} \bar{R}|_{C}^{2}\right)-\alpha\left(|\bar{S} \bar{P}|_{C}-|\bar{Q} \bar{R}|_{C}\right)^{2}=(1-\alpha)\left(|\bar{S} \bar{P}|_{C}^{2}-|\bar{Q} \bar{R}|_{C}^{2}\right)+2 \alpha|\bar{Q} \bar{R}|_{C}\left(|\bar{S} \bar{P}|_{C}-|\bar{Q} \bar{R}|_{C}\right) .
$$


Therefore, we obtain

$$
\begin{aligned}
\frac{1-\alpha}{2}\left(|\bar{Q} \bar{R}|_{C}^{2}-|\bar{S} \bar{P}|_{C}^{2}\right)+\alpha|\bar{Q} \bar{R}|_{C}\left(|\bar{Q} \bar{R}|_{C}-|\bar{S} \bar{P}|_{C}\right) \\
\leqslant|\bar{P} \bar{Q}|_{C}^{2}+|\bar{T} \bar{Q}|_{C}^{2}-|\bar{T} \bar{P}|_{C}^{2}+|\bar{S} \bar{R}|_{C}^{2}+|\bar{T} \bar{R}|_{C}^{2}-|\bar{T} \bar{S}|_{C}^{2} \\
\quad-\frac{1-\alpha}{2}\left(|\bar{R} \bar{S}|_{C}-|\bar{P} \bar{Q}|_{C}\right)^{2} \\
\leqslant|\bar{P} \bar{Q}|_{C}^{2}+|\bar{T} \bar{Q}|_{C}^{2}-|\bar{T} \bar{P}|_{C}^{2}+|\bar{S} \bar{R}|_{C}^{2}+|\bar{T} \bar{R}|_{C}^{2}-|\bar{T} \bar{S}|_{C}^{2} \\
\quad-\frac{1-\alpha}{2}\left(|\bar{R} \bar{S}|_{C}^{2}+|\bar{P} \bar{Q}|_{C}^{2}-\beta|\bar{R} \bar{S}|_{C}^{2}-\frac{1}{\beta}|\bar{P} \bar{Q}|_{C}^{2}\right) \\
=|\bar{P} \bar{Q}|_{C}^{2}\left(1-\frac{1-\alpha}{2}\left(1-\frac{1}{\beta}\right)\right)+|\bar{T} \bar{Q}|_{C}^{2}-|\bar{T} \bar{P}|_{C}^{2} \\
\quad+|\bar{S} \bar{R}|_{C}^{2}\left(1-\frac{1-\alpha}{2}(1-\beta)\right)+|\bar{T} \bar{R}|_{C}^{2}-|\bar{T} \bar{S}|_{C}^{2}
\end{aligned}
$$

for any $\beta>0$, where we have used $2|\bar{R} \bar{S}|_{C} \cdot|\bar{P} \bar{Q}|_{C} \leqslant \beta|\bar{R} \bar{S}|_{C}^{2}+\frac{1}{\beta}|\bar{P} \bar{Q}|_{C}^{2}$. By recalling the definition of the cone metric $|\cdot|_{C}$, we have

$$
\begin{aligned}
& |\bar{Q} \bar{R}|_{C}=2 \sin \frac{d_{Q R}}{2}, \quad|\bar{S} \bar{P}|_{C}=2 \sin \frac{d_{S P}}{2}, \\
& |\bar{P} \bar{Q}|_{C}=2 \sin \frac{d_{P Q}}{2}, \quad|\bar{R} \bar{S}|_{C}=2 \sin \frac{d_{R S}}{2}, \\
& |\bar{T} \bar{Q}|_{C}=|\bar{T} \bar{R}|_{C}=\frac{|\bar{Q} \bar{R}|_{C}}{2}=\sin \frac{d_{Q R}}{2}
\end{aligned}
$$

and (by noticing that $|O \bar{T}|_{C}=\cos \frac{d_{Q R}}{2}$,)

$$
\begin{aligned}
& |\bar{T} \bar{P}|_{C}^{2}=1+\cos ^{2} \frac{d_{Q R}}{2}-2 \cos \frac{d_{Q R}}{2} \cos d_{P Q_{m}}, \\
& |\bar{T} \bar{S}|_{C}^{2}=1+\cos ^{2} \frac{d_{Q R}}{2}-2 \cos \frac{d_{Q R}}{2} \cos d_{S Q_{m}} .
\end{aligned}
$$

Then

$$
\begin{aligned}
|\bar{T} \bar{Q}|_{C}^{2}-|\bar{T} \bar{P}|_{C}^{2} & =\sin ^{2} \frac{d_{Q R}}{2}-1-\cos ^{2} \frac{d_{Q R}}{2}+2 \cos \frac{d_{Q R}}{2} \cos d_{P Q_{m}} \\
& =2 \cos \frac{d_{Q R}}{2}\left(\cos d_{P Q_{m}}-\cos \frac{d_{Q R}}{2}\right) \\
& =2 \cos \frac{d_{Q R}}{2}\left(\cos d_{P Q_{m}}-\cos d_{Q Q_{m}}\right),
\end{aligned}
$$

where we have used $d_{Q Q_{m}}=\frac{d_{Q R}}{2}$. Similarly, we have

$$
|\bar{T} \bar{R}|_{C}^{2}-|\bar{T} \bar{S}|_{C}^{2}=2 \cos \frac{d_{Q R}}{2}\left(\cos d_{S Q_{m}}-\cos d_{R Q_{m}}\right) .
$$

Therefore, the combination of these and the estimate (2.3) implies the desired (2.2). The proof is completed.

\subsection{Harmonic maps.}

In the following, we always assume that $\Omega$ is a bounded domain in an $n$-dimensional smooth Riemannian manifold $(M, g)$ with $\operatorname{Ric}_{M} \geqslant-K$ for some $K \geqslant 0$ and that $\left(X, d_{X}\right)$ is a $C A T(\kappa)$ space for some $\kappa \geqslant 0$. 
Given any $\phi \in W^{1,2}(\Omega, X)$, we set

$$
W_{\phi}^{1,2}(\Omega, X):=\left\{u \in W^{1,2}(\Omega, X): d_{X}(u(x), \phi(x)) \in W_{0}^{1,2}(\Omega)\right\} .
$$

Using the variation method, it was proved in [28, 33] that there exists a unique $u \in W_{\phi}^{1,2}(\Omega, X)$ which is a minimizer of energy $E_{2}^{u}$ in $W_{\phi}^{1,2}(\Omega, X)$. That is, the energy $E^{u}:=E_{2}^{u}=E_{2}^{u}(\Omega)$ of $u$ satisfies

$$
E^{u}=\inf _{w}\left\{E^{w}: w \in W_{\phi}^{1,2}(\Omega, X)\right\} .
$$

Such an energy minimizing map is called a harmonic map.

The basic existence and regularity were given by Korevaar-Schoen in [31] for $\kappa \leqslant 0$ and by Serbinowski in [43] for $\kappa>0$. We state their regularity result in the case $\kappa>0$ (see also [36, Theorem 2.3]):

Theorem 2.4 ([31, 43]). Let $u$ be a harmonic map from $\Omega$ to $X$. Assume that its image $u(\Omega)$ is contained in a ball $B_{\rho} \subset X$ with radius $\rho<\frac{\pi}{2 \sqrt{\kappa}}$ if $\kappa>0$. Then $u$ is locally Lipschitz continuous in the interior of $\Omega$. (Note that the local Lipschitz constant of $u$ near a point $x \in \Omega$ depends on the $C^{1}$-norm of metric g near $x$.)

We need also the following property:

Lemma 2.5. (Serbinowski [43, Proposition 1.17], Fuglede [15, Lemma 2]). Let $\kappa>0$. Assume that its image $u(\Omega)$ is contained in a ball $B_{\rho}(P) \subset X$ with radius $\rho<\frac{\pi}{2 \sqrt{\kappa}}$. Then the function $f_{P}(x):=\cos \left(\sqrt{\kappa} \cdot d_{X}(u(x), P)\right)$ satisfies $f_{P} \in W^{1,2}(\Omega)$ and

$$
\Delta f_{P} \leqslant-\kappa \cdot f_{P} \cdot e_{u}
$$

in the sense of distributions. If $\kappa=0$, then for any $P \in X$ we have $\Delta d_{X}^{2}(P, u(x)) \geqslant 2 e_{u}$ in the sense of distributions.

Recall that

$$
\operatorname{Lip} u(x)=\limsup _{y \rightarrow x} \frac{d_{X}(u(x), u(y))}{|x y|}=\limsup _{r \rightarrow 0} \sup _{y \in B_{x}(r)} \frac{d_{X}(u(x), u(y))}{r} .
$$

The above lemma implies the following point-wise estimates, which is a corollary of the mean value inequality for subharmonic functions.

Corollary 2.6. Let $u$ be a harmonic map from $\Omega$ to $X$. Assume that its image $u(\Omega)$ is contained in a ball $B_{\rho} \subset X$ with radius $\rho<\frac{\pi}{2 \sqrt{\kappa}}$ if $\kappa>0$. Then there exists a constant $C=C(n, \sqrt{K} R)$ depending only on $n$ and $\sqrt{K} R$ such that: for any ball $B_{R}$ with $B_{2 R} \subset \subset \Omega$, we have

$$
\operatorname{Lip}^{2} u(x) \leqslant C \cdot e_{u}(x), \quad \text { for almost all } x \in B_{R / 6} .
$$

Proof. For the case $\kappa=0$, this is Theorem 5.5 in [47]. We need only to show the assertion for the case $\kappa>0$. Without loss of the generality, we can assume $\kappa=1$ in this case. The argument is similar to the proof of Theorem 5.5 in [47].

(i). Fix any $z$ with $B_{2 R}(z) \subset \subset \Omega$. From the continuity of $u$, there exists a small neighborhood $O$ of $z$ such that $\operatorname{diam} u(O)<\pi / 2$ and $O \subset B_{R}(z)$, where $\operatorname{diam} u(O)$ is the diameter of $u(O)$.

By using Lemma 2.5 and the fact that $\left|\nabla d_{X}(u(x), P)\right| \leqslant e_{u}$ for any fixed $P \in X$, it is easy the check that $\Delta d_{X}\left(u(x), u\left(y_{0}\right)\right) \geqslant 0$ on $O$ for any fixed $y_{0} \in O$, in the sense of distributions. Let $\Delta^{(2)}$ be the Laplace-Beltrami operator on $M \times M$, the product manifold (with the product metric and the product measure). Consider the function $\rho_{u}:=d_{X}(u(x), u(y))$ on $O \times O$. Hence, we obtain

$$
\Delta^{(2)} \rho_{u}(x, y) \geqslant 0 \quad \text { on } \quad O \times O
$$


in the sense of distributions (see the step (iii) in the proof of [47, Proposition 5.4] for the details).

From the mean value inequality for subharmonic functions on $O \times O$ (see [40, Theorem 6.2 of Chapter II]), we conclude that, for any ball $B_{r}\left(\left(z_{1}, z_{2}\right)\right)$ with $B_{2 r}\left(\left(z_{1}, z_{2}\right)\right) \subset \subset O \times O$,

$$
\begin{aligned}
\sup _{(x, y) \in B_{r}\left(\left(z_{1}, z_{2}\right)\right)} \rho_{u}^{2}(x, y) & \leqslant C_{1} 2^{C_{2}(1+\sqrt{K} r)} \cdot f_{B_{2 r}\left(\left(z_{1}, z_{2}\right)\right)} \rho_{u}^{2}(x, y) d v_{g}(x) d v_{g}(y) \\
& \leqslant C_{3}(n, \sqrt{K} R) \cdot f_{B_{2 r}\left(\left(z_{1}, z_{2}\right)\right)} \rho_{u}^{2}(x, y) d v_{g}(x) d v_{g}(y),
\end{aligned}
$$

where the constants $C_{1}, C_{2}$ depend only on $n$, and $C_{3}(n, \sqrt{K} R)=C_{1} 2^{C_{2}(1+\sqrt{K} R)}$.

(ii). Since $B_{2 r}((z, z)) \subset B_{2 r}(z) \times B_{2 r}(z)$, the Poincaré inequality for $W^{1,2}(\Omega, X)$-maps (see [32], and also Proposition 2.1 (3) and Remark 2.1) states that the RHS of (2.6) for $z_{1}=z_{2}=z$ can be dominated by $C_{4}(n, \sqrt{K} R) \cdot r^{n+2} \int_{B_{12 r}(z)} e_{u}(x) \mathrm{d} v_{g}(x)$. Therefore, we have

$$
\begin{aligned}
\sup _{y \in B_{r}(z)} \frac{\rho_{u}^{2}(z, y)}{r^{2}} & \leqslant \sup _{(x, y) \in B_{r}((z, z))} \frac{\rho_{u}^{2}(x, y)}{r^{2}} \\
& \leqslant C_{3} C_{4} \cdot \frac{r^{n} \cdot \operatorname{vol}\left(B_{z}(12 r)\right)}{\operatorname{vol}\left(B_{2 r}((z, z)) \subset \Omega \times \Omega\right)} f_{B_{12 r}(z)} e_{u}(x) d v_{g}(x) \\
& \leqslant C_{5}(n, \sqrt{K} R) \cdot f_{B_{12 r}(z)} e_{u}(x) d v_{g}(x),
\end{aligned}
$$

where we have used Bishop-Gromov inequality and $\operatorname{vol}\left(B_{2 r}((z, z))\right) \geqslant \operatorname{vol}^{2}\left(B_{r}(z)\right)$.

Notice that $\lim _{r \rightarrow 0} f_{B_{12 r}(z)} e_{u}(x) d v_{g}(x)=e_{u}(z)$ for almost all $z \in B_{R / 6}$ and that $\operatorname{Lip} u(z)=$ $\lim \sup _{r \rightarrow 0} \sup _{y \in B_{r}(z)} \rho_{u}(y, z) / r$. By letting $r \rightarrow 0$ in (2.7), it follows the desired estimate.

\subsection{Generalized Rademacher theorem for Lipschitz maps.}

Let $\Omega$ be a bounded domain of an $n$-dimensional Riemannian manifold $(M, g)$. Recall that the classical Rademacher theorem states that any Lipschitz function $f: \Omega \rightarrow \mathbb{R}$ is differentiable at almost all $x \in \Omega$.

For our purpose, we have to consider the differentiability of maps into a metric space $\left(X, d_{X}\right)$. Let us recall the notion of metric differential for maps from $\Omega$ into a metric space, which was introduced by Kirchheim in [30].

Definition 2.7. We say that a map $f: \Omega \rightarrow X$ is metrically differentiable at $x_{0}$ if there exists a semi-norm $\|\cdot\|_{x_{0}}$ in $T_{x_{0}} M:=\mathbb{R}^{n}$ such that

$$
d_{X}\left(f\left(\exp _{x_{0}}(t \xi)\right), f\left(x_{0}\right)\right)-t \cdot\|\xi\|_{x_{0}}=o(t),
$$

for all $\xi \in \mathbb{S}^{n-1} \subset T_{x_{0}} M$. This semi-norm will be called the metric differential and be denoted by $m d f_{x_{0}}$.

The following generalized Rademacher's theorem for maps was given in [30].

Theorem 2.8 (Kirchheim [30]). Any Lipschitz map $f: \Omega \rightarrow X$ is metrically differentiable at almost all $x \in \Omega$.

If a Lipschitz continuous map $f: \Omega \rightarrow X$ is metrically differentiable at $x$, we put

$$
G_{f}(x):=\max _{\xi \in \mathbb{S}^{n-1}} m d f_{x}(\xi) .
$$

Lemma 2.9. Let $f: \Omega \rightarrow X$ be a Lipschitz function. If $f$ is metrically differentiable at $x$, then we have

$$
G_{f}(x)=\operatorname{Lip} f(x)
$$


Proof. From the definition of $G_{f}(x)$, it is clear that $G_{f}(x) \leqslant \operatorname{Lip} f(x)$.

For the converse, we choose a sequence of points $\left\{y_{j}:=\exp _{x}\left(t_{j} \xi_{j}\right)\right\}_{j=1}^{\infty} \subset \Omega$ such that $\lim _{j \rightarrow \infty} t_{j}=0,\left|\xi_{j}\right|=1$, and

$$
\operatorname{Lip} f(x)=\lim _{j \rightarrow \infty} \frac{d_{X}\left(f\left(y_{j}\right), f(x)\right)}{t_{j}} .
$$

Since $f$ is metrically differentiable at $x$, we have

$$
d_{X}\left(f\left(y_{j}\right), f(x)\right)=m d f_{x}\left(\xi_{j}\right) \cdot t_{j}+o\left(t_{j}\right),
$$

From the definition of $G_{f}(x)$, we have

$$
\operatorname{Lip} f(x)=\lim _{j \rightarrow \infty} m d f_{x}\left(\xi_{j}\right) \leqslant G_{f}(x) .
$$

The proof is complete.

\section{AN ASYMPTOTIC MEAN VALUE INEQUALITY}

We will consider some asymptotic behaviors of harmonic maps from a domain of smooth Riemannian manifold to a $C A T(1)$-space. Let us begin with the following mean value property, which is similar to Proposition 2.1 of Chapter I in [40].

Lemma 3.1. Let $(M, g)$ be an n-dimensional Riemannian manifold with Ric $_{M} \geqslant-K$ for some $K \in \mathbb{R}$. Suppose that $f$ is a Lipschitz function on an open subset $\Omega \subset M, f \geqslant 0$, and $\Delta f \leqslant g \in$ $L_{\mathrm{loc}}^{1}(\Omega)$ in the sense of distributions. Then for any $p \in \Omega$ and $R>0$ with $B_{R}(p) \subset \subset \Omega$,

$$
\frac{1}{A_{K}(R)} \int_{\partial B_{R}(p)} f \leqslant f(p)+\int_{0}^{R} \frac{\int_{B_{r}(p)} g(x) \mathrm{d} v_{g}(x)}{A_{K}(r)} d r,
$$

where $A_{K}(r)$ is the area of a geodesic sphere of radius $r$ in the simply connected space form of constant curvature $-K /(n-1)$.

Proof. Since $\Delta f \leqslant g$, we have by divergence theorem that

$$
\begin{aligned}
\int_{B_{r}(p)} g(x) \mathrm{d} v_{g}(x) & \geqslant \int_{B_{r}(p)} \Delta f \mathrm{~d} v_{g}=\int_{\partial B_{r}(p)} \frac{\partial f}{\partial r} \\
& =\frac{\partial}{\partial r} \int_{\partial B_{r}(p)} f-\int_{\partial B_{r}(p)} H f,
\end{aligned}
$$

where $0<r<R$, and $H$ is the mean curvature of $\partial B_{r}(p)$ with resect to $\partial / \partial r$. The standard comparison theorem asserts that

$$
H(x) \leqslant(n-1) \cot _{K}(r)=\frac{A_{K}^{\prime}(r)}{A_{K}(r)}, \quad \forall x \in \partial B_{r}(p) .
$$

Therefore, it follows from (3.2) and the assumption $f \geqslant 0$ that

$$
\frac{\int_{B_{r}(p)} g(x) \mathrm{d} v_{g}(x)}{A_{K}(r)} \geqslant \frac{\partial}{\partial r} \frac{\int_{\partial B_{r}(p)} f}{A_{K}(r)} .
$$

Notice that $\lim _{r \rightarrow 0} \frac{\int_{\partial B_{r}(p)} f}{A_{K}(r)}=f(p)$. Integrating both sides of the above inequality with respect to $r$ over $(0, R)$, we conclude that 3.1 holds.

Now we consider the case that $f$ needs not to be nonnegative. 
Corollary 3.2. Let $(M, g)$ be an $n$-dimensional Riemannian manifold with Ric $_{M} \geqslant-K$ for some $K \in \mathbb{R}$. Suppose that $f$ is a Lipschitz function on an open subset $\Omega \subset M$, and $\Delta f \leqslant g \in L_{\mathrm{loc}}^{1}(\Omega)$ in the sense of distributions. Then for any $p \in \Omega$ and $R>0$ with $B_{R}(p) \subset \subset \Omega$,

$$
\begin{aligned}
\frac{1}{V_{K}(R)} \int_{B_{R}(p)}(f(x)-f(p)) \mathrm{d} v_{g}(x) \leqslant & R \cdot \operatorname{Lip}_{B_{R}(p)} f \cdot\left(1-\frac{\operatorname{vol}\left(B_{R}(p)\right)}{V_{K}(R)}\right) \\
& +\frac{1}{V_{K}(R)} \int_{0}^{R} A_{K}(r) \int_{0}^{r} \frac{\int_{B_{s}(p)} g(x) \mathrm{d} v_{g}(x)}{A_{K}(s)} \mathrm{d} s \mathrm{~d} r .
\end{aligned}
$$

where $V_{K}(r)$ is the is the volume of a geodesic ball of radius $r$ in the space form of constant curvature $-K /(n-1)$, and

$$
\operatorname{Lip}_{B_{R}(p)} f:=\sup _{x, y \in B_{R}(p)} \frac{|f(x)-f(y)|}{|x y|} .
$$

In particular, if p is a Lebesgue point of $g$, then the following asymptotic mean value inequality holds

$$
\frac{1}{V_{K}(R)} \int_{B_{R}(p)}(f(x)-f(p)) \mathrm{d} v_{g}(x) \leqslant \frac{g(p)}{2(n+2)} \cdot R^{2}+o\left(R^{2}\right) \quad \text { as } R \rightarrow 0 .
$$

Proof. We consider the function $h(x):=f(x)-f(p)$. By applying Lemma 3.1 to nonnegative function

$$
h_{r}(x):=h(x)-\inf _{y \in B_{r}(p)} h(y)
$$

on $B_{r}(p), 0<r<R$, we have

$$
\frac{1}{A_{K}(r)} \int_{\partial B_{r}(p)} h_{r} \leqslant h_{r}(p)+G_{K}(r)=-\inf _{y \in B_{r}(p)} h(y)+G_{K}(r)
$$

where

$$
G_{K}(r):=\int_{0}^{r} \frac{\int_{B_{s}(p)} g(x) \mathrm{d} v_{g}(x)}{A_{K}(s)} d s .
$$

Denote by $A(r):=\operatorname{vol}_{n-1}\left(\partial B_{r}(P) \subset M\right)$. We get

$$
\int_{\partial B_{r}(p)} h \leqslant-\inf _{y \in B_{r}(p)} h(y) \cdot\left(A_{K}(r)-A(r)\right)+G_{K}(r) \cdot A_{K}(r) .
$$

Remark that $h(p)=0$ and $\operatorname{Lip}_{B_{r}(p)} h \leqslant \operatorname{Lip}_{B_{R}(p)} h=\operatorname{Lip}_{B_{R}(p)} f$, so we have

$$
-\inf _{y \in B_{r}(p)} h(y) \leqslant-\inf _{y \in B_{R}(p)} h(y) \leqslant R \cdot \operatorname{Lip}_{B_{R}(p)} f .
$$

The above two inequalities implies

$$
\int_{\partial B_{r}(p)} h \leqslant R \cdot \operatorname{Lip}_{B_{R}(p)} f \cdot\left(A_{K}(r)-A(r)\right)+G_{K}(r) \cdot A_{K}(r) .
$$

where we have used the Bishop inequality $A(r) \leqslant A_{K}(r)$ for all $r \in(0, R)$. Integrating both sides of the above inequality with respect to $r$ over $(0, R)$, and then dividing by $V_{K}(R)$, we get (3.3).

Suppose that $p$ is a Lebesgue point of $g$, i.e.,

$$
\lim _{R \rightarrow 0} f_{B_{R}(p)} g(x) \mathrm{d} v_{g}(x)=g(p) .
$$

Notice that

$$
\frac{\operatorname{vol}\left(B_{R}(p)\right)}{V_{K}(R)}=1+O\left(R^{2}\right) \quad \text { as } R \rightarrow 0 .
$$


It follows that

$$
R \cdot \operatorname{Lip}_{B_{R}(p)} f \cdot\left(1-\frac{\operatorname{vol}\left(B_{R}(p)\right)}{V_{K}(R)}\right)=O\left(R^{3}\right) \quad \text { as } R \rightarrow 0
$$

and that

$$
\frac{1}{V_{K}(R)} \int_{B_{R}(p)} g(x) \mathrm{d} v_{g}(x)=g(p)+o(1) \quad \text { as } R \rightarrow 0 .
$$

Thus, by a direct calculation (noticing that $A_{K}(t)=\omega_{n-1} \cdot t^{n-1}+O\left(t^{n}\right)$ as $t \rightarrow 0$ ), we get

$$
\begin{aligned}
& \frac{1}{V_{K}(R)} \int_{0}^{R} A_{K}(r) \int_{0}^{r} \frac{\int_{B_{s}(p)} g(x) \mathrm{d} v_{g}(x)}{A_{K}(s)} \mathrm{d} s \mathrm{~d} r \\
& =\frac{1}{V_{K}(R)} \int_{0}^{R} A_{K}(r) \int_{0}^{r} \frac{V_{K}(s) \cdot(g(p)+o(1))}{A_{K}(s)} \mathrm{d} s \mathrm{~d} r \\
& =\frac{1}{V_{K}(R)} \int_{0}^{R} A_{K}(r) \int_{0}^{r} \frac{s}{n}(1+o(1))(g(p)+o(1)) \mathrm{d} s \mathrm{~d} r \\
& =\frac{g(p)}{2(n+2)} \cdot R^{2}+o\left(R^{2}\right)
\end{aligned}
$$

as $R \rightarrow 0$. The proof is finished.

At last in this section, we want to use the above asymptotic mean value inequality to harmonic maps to a metric space with curvature bounded above.

Lemma 3.3. Let $\Omega$ be a bounded domain in an $n$-dimensional smooth Riemannian manifold $(M, g)$ and that $\left(X, d_{X}\right)$ is a CAT(1)-space. Suppose that $u$ is a harmonic map from $\Omega$ to $X$. Given any $a, b \in \mathbb{R}$ with $a, b \geqslant 0$, and any $q \in \Omega, Q \in X$, we put

$$
w_{a, b, Q, q}(x):=a \cdot d_{X}^{2}(u(x), u(q))+b \cdot \cos \left(d_{X}(u(x), Q)\right) .
$$

Then there exists a sequence $\left\{\epsilon_{j}\right\}_{j \in \mathbb{N}}$ with $\epsilon_{j} \rightarrow 0$ as $j \rightarrow \infty$ and a subset $\mathscr{N} \subset \Omega$ with zero measure such that

$$
\begin{aligned}
\int_{B_{\epsilon_{j}}(0)} & {\left[w_{a, b, P, x_{0}}\left(\exp _{x_{0}}(\eta)\right)-w_{a, b, P, x_{0}}\left(x_{0}\right)\right] \mathrm{d} \eta } \\
& \leqslant\left(2 a-b \cdot \cos \left(d_{X}\left(u\left(x_{0}\right), P\right)\right)\right) \frac{\omega_{n-1}}{2 n(n+2)} \cdot e_{u}\left(x_{0}\right) \cdot \epsilon_{j}^{n+2}+o\left(\epsilon_{j}^{n+2}\right)
\end{aligned}
$$

for any $x_{0} \in \Omega \backslash \mathscr{N}$ and for any $P \in X$ such that the image $u(\Omega)$ is contained in a ball $B_{\rho}(P) \subset X$ with radius $\rho<\frac{\pi}{2}$, and for every $a, b \geqslant 0$.

Proof. Recall that $e_{2, \epsilon}^{u} \rightarrow e_{u}$ in $L_{\text {loc }}^{1}(\Omega)$ as $\epsilon \rightarrow 0$. Therefore, there exists a sequence $\left\{\epsilon_{j}\right\}_{j \in \mathbb{N}}$ with $\epsilon_{j} \rightarrow 0$ as $j \rightarrow \infty$ such that

$$
\lim _{j \rightarrow \infty} e_{2, \epsilon_{j}}^{u}\left(x_{0}\right)=e_{u}\left(x_{0}\right) \quad \text { for almost all } x_{0} \in \Omega
$$

By the definition of the approximating energy density, it follows that

$$
\int_{B_{\epsilon_{j}}\left(x_{0}\right)} d_{X}^{2}\left(u(x), u\left(x_{0}\right)\right) \mathrm{d} v_{g}(x)=\frac{\omega_{n-1}}{n(n+2)} \cdot e_{u}\left(x_{0}\right) \cdot \epsilon_{j}^{n+2}+o\left(\epsilon_{j}^{n+2}\right)
$$


for almost all point $x_{0} \in \Omega$. On the other hand, we have by Lemma 2.5 and Corollary 3.2 that

$$
\begin{aligned}
\int_{B_{\epsilon_{j}}\left(x_{0}\right)}[ & \left.\cos \left(d_{X}(u(x), P)\right)-\cos \left(d_{X}\left(u\left(x_{0}\right), P\right)\right)\right] \mathrm{d} v_{g}(x) \\
& \leqslant V_{K}\left(\epsilon_{j}\right) \cdot\left[-\frac{\cos \left(d_{X}\left(u\left(x_{0}\right), P\right)\right)}{2(n+2)} \cdot e_{u}\left(x_{0}\right) \cdot \epsilon_{j}^{2}+o\left(\epsilon_{j}^{2}\right)\right] \\
& =-\frac{\cos \left(d_{X}\left(u\left(x_{0}\right), P\right)\right) \cdot \omega_{n-1}}{2 n(n+2)} \cdot e_{u}\left(x_{0}\right) \cdot \epsilon_{j}^{n+2}+o\left(\epsilon_{j}^{n+2}\right)
\end{aligned}
$$

for all Lebesgue points $x_{0}$ of $e_{u}$, and for all $P \in X$ such that the image $u(\Omega)$ is contained in a ball $B_{\rho}(P) \subset X$ with radius $\rho<\frac{\pi}{2}$. Here we have used that $V_{K}\left(\epsilon_{j}\right)=\frac{\omega_{n-1}}{n} \cdot \epsilon_{j}^{n}+o\left(\epsilon_{j}^{n}\right)$. Thus, for almost all $x_{0} \in \Omega$, we have

$$
\begin{aligned}
\int_{B_{\epsilon_{j}}\left(x_{0}\right)} & {\left[w_{a, b, P, x_{0}}(x)-w_{a, b, P, x_{0}}\left(x_{0}\right)\right] \mathrm{d} v_{g}(x) } \\
& \leqslant\left(2 a-b \cdot \cos \left(d_{X}\left(u\left(x_{0}\right), P\right)\right)\right) \frac{\omega_{n-1}}{2 n(n+2)} \cdot e_{u}\left(x_{0}\right) \cdot \epsilon_{j}^{n+2}+o\left(\epsilon_{j}^{n+2}\right)
\end{aligned}
$$

and any $P \in X$, and for every $a, b \in \mathbb{R}$ with $b \geqslant 0$.

At last, we consider the exponential map $\exp _{x_{0}}: B_{\epsilon_{j}}(0) \subset \mathbb{R}^{n} \rightarrow B_{\epsilon_{j}}\left(x_{0}\right)$. It is well known

$$
\frac{\mathrm{d} v_{g}}{\mathrm{~d} \eta}=1+o\left(\epsilon_{j}\right)
$$

Thus, for any general Lipschitz function $h$, we have, as $r \rightarrow 0$,

$$
\begin{aligned}
\int_{B_{r}(0)} & \left(h\left(\exp _{x_{0}}(\eta)\right)-h\left(x_{0}\right)\right) \mathrm{d} \eta \\
& \leqslant \int_{B_{r}\left(x_{0}\right)}\left(h(x)-h\left(x_{0}\right)\right)(1+o(r)) \mathrm{d} v_{g}(x) \\
& \leqslant \int_{B_{r}\left(x_{0}\right)}\left(h(x)-h\left(x_{0}\right)\right) \mathrm{d} v_{g}(x)+o(r) \cdot \int_{B_{r}\left(x_{0}\right)}\left|h(x)-h\left(x_{0}\right)\right| \mathrm{d} v_{g}(x) \\
& \leqslant \int_{B_{r}\left(x_{0}\right)}\left(h(x)-h\left(x_{0}\right)\right) \mathrm{d} v_{g}(x)+o(r) \cdot O(r) \cdot O\left(r^{n}\right)
\end{aligned}
$$

By using this to $w_{a, b, P, x_{0}}$ and combining with (3.9), we obtain Eq. (3.8).

\section{The BochNer INEQUALITY FOR HARMONIC MAPS INTO $C A T(\kappa)$-SPACES}

Let $\Omega$ be a bounded domain in an $n$-dimensional smooth Riemannian $(M, g)$ with $\operatorname{Ric}_{M} \geqslant-K$ for some $K \geqslant 0$, and let $\left(X, d_{X}\right)$ be a complete $C A T(\kappa)$-space for some $\kappa>0$.

In this section, we always assume that $u: \Omega \rightarrow X$ is a harmonic map with the image Im(u) containing in a ball $B_{\rho}\left(Q_{0}\right) \subset Y$ with $\rho<\pi /(2 \sqrt{\kappa})$. From Theorem 2.4 we know that $u$ is local Lipschitz continuous on $\Omega$.

\subsection{Auxiliary functions.}

In this subsection, we will introduce a family of auxiliary functions.

Fix $p \in(1, \infty)$ and a ball $B_{R}(o)$ such that $B_{2 R}(o) \subset \subset \Omega$. Denote by $B_{R}:=B_{R}(o)$ and by

$$
\ell_{0}:=\operatorname{Lip}_{B_{2 R}} u=\sup _{x, y \in B_{2 R}, x \neq y} \frac{d_{X}(u(x), u(y))}{|x y|}<\infty .
$$


We introduce a family of auxiliary functions $f_{t}(x)$ on $B_{R}$ as follows: for any $t>0$, we define

$$
f_{t}(x):=\inf _{y \in B_{2 R}}\left\{\frac{|x y|^{p}}{p t^{p-1}}-F(x, y)\right\}, \quad x \in B_{R},
$$

where

$$
F(x, y):=2 \sin \frac{d_{X}(u(x), u(y))}{2}+4 \sin ^{2} \frac{d_{X}(u(x), u(y))}{2} .
$$

It is clear that $F(x, y) \leqslant 6$ and that (by taking $y=x$ )

$$
0 \geqslant f_{t}(x) \geqslant-6 \text {. }
$$

For any $0<t<t_{*}\left(:=\left(R^{p} / 6 p\right)^{1 /(p-1)}\right)$, it is clear that the the "inf" of (4.1) can be achieved, i.e., for any $x \in B_{R}$,

$$
S_{t}(x):=\left\{y \in B_{2 R} \mid f_{t}(x)=\frac{|x y|^{p}}{p t^{p-1}}-F(x, y)\right\} \neq \varnothing .
$$

Since $F(x, \cdot)$ is continuous on $B_{2 R}$, it follows that $S_{t}(x)$ is close. Fix any small $t \in\left(0, t_{*}\right)$. We define two functions on $B_{R}$

$$
L_{t}(x):=\min _{y \in S_{t}(x)}|x y| \quad \text { and } \quad D_{t}(x):=\frac{L_{t}^{p}(x)}{p t^{p-1}}-f_{t}(x) .
$$

We give some basic properties of these functions.

Lemma 4.1. For any $t \in\left(0, t_{*}\right)$, we have the following properties.

(1) $f_{t}$ is Lipschitz continuous on $B_{R}$;

(2) Both $L_{t}$ and $D_{t}$ are lower semi-continuous on $B_{R}$;

(3) There exists a constant $C=C\left(p, \ell_{0}, \kappa\right)>0$ such that, for any $t \in\left(0, t_{*}\right)$,

$$
L_{t} \leqslant C t, \quad D_{t} \leqslant C t \quad \text { and } \quad-f_{t} \leqslant C t \quad \text { on } B_{R} .
$$

Proof. (1) Take any $x_{1}, x_{2} \in B_{R}$. From the definition of $f_{t}$, we by choosing some $y_{2} \in S_{t}\left(x_{2}\right)$ have that

$$
f_{t}\left(x_{1}\right)-f_{t}\left(x_{2}\right) \leqslant \frac{\left|x_{1} y_{2}\right|^{p}-\left|x_{2} y_{2}\right|^{p}}{p t^{p-1}}-\left(F\left(x_{1}, y_{2}\right)-F\left(x_{2}, y_{2}\right)\right) .
$$

Noticing that both $|\cdot \cdot|^{p}$ and $F(\cdot, \cdot)$ are Lipschitz, we conclude that there exists some constant $C_{t}>0$ such that $f_{t}\left(x_{1}\right)-f_{t}\left(x_{2}\right) \leqslant C_{t}\left|x_{1} x_{2}\right|$. That is, $f_{t}$ is Lipschitz continuous.

(2) From the definition of $L_{t}$, we know that $L_{t}$ is lower semi-continuous. By using that $f_{t}$ is continuous, we get that $D_{t}$ is also lower semi-continuous.

(3) We take some $y_{t} \in S_{t}(x)$ such that $L_{t}(x)=\left|x y_{t}\right|$. We have

$$
\begin{aligned}
D_{t}(x) & =\frac{L_{t}^{p}(x)}{p t^{p-1}}-f_{t}(x)=F\left(x, y_{t}\right) \leqslant d_{X}\left(u(x), u\left(y_{t}\right)\right)+d_{X}^{2}\left(u(x), u\left(y_{t}\right)\right) \\
& \leqslant(1+\pi / \sqrt{\kappa}) \cdot d_{X}\left(u(x), u\left(y_{t}\right)\right) \leqslant(1+\pi / \sqrt{\kappa}) \cdot \ell_{0} \cdot L_{t}(x) \\
& :=C_{1}\left(\ell_{0}, \kappa\right) L_{t}(x),
\end{aligned}
$$

where we have used

$$
d_{X}\left(u(x), u\left(y_{t}\right)\right) \leqslant d_{X}\left(u(x), Q_{0}\right)+d_{X}\left(Q_{0}, u\left(y_{t}\right)\right)<\pi / \sqrt{\kappa}
$$

Noticing that $f_{t} \leqslant 0$, we get $D_{t} \geqslant \frac{L_{t}^{p}(x)}{p t^{p-1}}$. By combining with (4.4), we have

$$
L_{t} \leqslant\left(p \cdot C_{1}\right)^{1 /(p-1)} \cdot t:=C_{2}\left(p, \ell_{0}, \kappa\right) \cdot t .
$$

By using (4.4) again, we get $D_{t} \leqslant C_{1} C_{2} \cdot t$. At last, $f_{t}=L_{t}^{p} /\left(p t^{p-1}\right)-D_{t} \geqslant-D_{t} \geqslant-C_{1} C_{2} \cdot t$. The proof is finished. 
The main result in this subsection is the following elliptic inequality for $f_{t}$.

Proposition 4.2. Assume that $\kappa=1, p \in[2, \infty)$ and $\operatorname{diam}\left(u\left(B_{2 R}\right)\right)<\pi / 2$, where $\operatorname{diam}\left(u\left(B_{2 R}\right)\right)$ is the diameter of the $u\left(B_{2 R}\right)$. Given any $\varepsilon>0$, we have that, for any $t>0$ sufficiently small, the inequality holds

$$
\Delta f_{t}(x) \leqslant \frac{K}{t^{p-1}} \cdot L_{t}^{p}(x)+(1+\varepsilon) \cdot e_{u}(x) D_{t}(x), \quad \text { on } B_{R},
$$

in the sense of distributions.

In order to prove this lemma, we need the following:

Lemma 4.3 (Perturbation Lemma). Let $U \subset M$ be a convex domain of $M$ and let $h \in W^{1,2}(U) \cap$ $C(U)$ satisfy $\Delta h \leqslant \lambda$ on $U$ for some constant $\lambda \in \mathbb{R}$. Assume that point $\hat{x} \in U$ is one of minimum points of function $h$ on $U$. Assume a subset $A \subset U$ has full measure.

Then for any $r, \delta>0$ sufficiently small, (they are smaller than a constant $\delta_{0}$ depending on the bounds of sectional curvature on $U$,) there exists a smooth function $\phi$ on a neighborhood of $\hat{x}$, $B_{r_{0}}(\hat{x})$, such that $h+\phi$ has a local minimum point in $B_{r}(\hat{x}) \cap A$ and that

$$
|\phi|+|\nabla \phi|+|\operatorname{Hess}(\phi)| \leqslant \delta, \quad \forall x \in B_{r_{0}}(\hat{x}) .
$$

Proof. This comes from a slight extension of the classical Jensen's lemma [6, Lemma A.3]. We will give the details of the proof in the Appendix A.

Proof of Proposition 4.2 Denote $\rho_{0}:=\operatorname{diam}\left(u\left(B_{2 R}\right)\right)$. It suffices to prove the following claim. Claim: There exists $\bar{t}=\bar{t}\left(p, \varepsilon, t_{*}, \pi / 2-\rho_{0}\right)$ such that for each $t \in(0, \bar{t})$, the function $f_{t}(\cdot)$ satisfies

$$
\Delta f_{t}(x) \leqslant \frac{K}{t^{p-1}} \cdot L_{t}^{p}(x)+(1+\varepsilon) e_{u}(x) D_{t}(x)+\theta \quad \text { on } B_{R}
$$

for any $\theta \in(0,1)$, in the sense of distributions.

We shall prove this Claim by a contradiction argument. Suppose that the Claim fails for some sufficiently small $t \in\left(0, t_{*}\right)$ and some $\theta_{0} \in(0,1)$. According to the maximum principle, there exists a domain $U \subset \subset B_{R}$ such that the function $f_{t}(\cdot)-v(\cdot)$ satisfies

$$
\min _{x \in U}\left(f_{t}(x)-v(x)\right)<0=\min _{x \in \partial U}\left(f_{t}(x)-v(x)\right),
$$

where $v$ is the (unique) solution of the Dirichlet problem

$$
\left\{\begin{array}{l}
\Delta v=\frac{K}{t^{p-1}} \cdot L_{t}^{p}(x)+(1+\varepsilon) e_{u}(x) D_{t}(x)+\theta_{0} \text { in } U \\
v \quad=f_{t} \quad \text { on } \partial U .
\end{array}\right.
$$

This means that $f_{t}(\cdot)-v(\cdot)$ has a strict minimum in the interior of $U$.

Let us define a function $H(x, y)$ on $U \times B_{2 R}$, by

$$
H(x, y):=\frac{|x y|^{p}}{p t^{p-1}}-F(u(x), u(y))-v(x) .
$$

Let $\bar{x} \in U$ be a minimum of $f_{t}(\cdot)-v$ on $U$, and let $\bar{y}:=y_{t}(\bar{x}) \in S_{t}(\bar{x})$ such that $L_{t}(\bar{x})=|\bar{x} \bar{y}|$. By the definition of $f_{t}$, we conclude that $H(x, y)$ has a minimum at $(\bar{x}, \bar{y})$.

Let $\mathscr{A} \subset U \times B_{2 R}$ be the set of all points $\left(x^{o}, y^{o}\right) \in U \times B_{2 R}$ satisfying the following two properties:

1) $x^{o} \neq y^{o}$, and $x^{o} \notin \mathscr{N}, y^{o} \notin \mathscr{N}$, where the set $\mathscr{N}$ is given as in Lemma3.3,

2) point $x^{o}$ is a Lebesgue point of $\frac{K}{t^{p-1}} \cdot L_{t}^{p}(x)+(1+\varepsilon) e_{u}(x) D_{t}(x)$.

It is clear that $\left(U \times B_{2 R}\right) \backslash \mathscr{A}$ has zero measure. 
Noting that the function $f(t)=2 \sin t+4 \sin ^{2} t$ satisfies $f^{\prime}(t)>0, f^{\prime \prime}(t)>0$ for $t<\pi / 6$ and (seeing the proof of Corollary 2.6)

$$
\Delta^{(2)} d_{X}(u(x), u(y)) \geqslant 0,
$$

we have $\Delta^{(2)} F(x, y) \geqslant 0$, where $\Delta^{(2)}$ is the Laplace-Beltrami operator on the product manifold $M \times M$. The Laplacian comparison theorem on product space $M \times M$ implies that $\Delta^{(2)}\left(|x y|^{2}\right) \leqslant$ $C_{n, K \text {,diam }(U)}$ for some constant $C_{n, K, \operatorname{diam}(U)}>0$. By using the assumption $p \geqslant 2$, we obtain $\Delta^{(2)}\left(|x y|^{p}\right) \leqslant C_{n, K, p, \operatorname{diam}(U)}$. Then, by Lemma 4.3, we conclude that, for any sufficiently small $\delta>0$, there exists a smooth function $\gamma_{\delta}(x, y)$ such that $\left|\gamma_{\delta}\right|+\left|\nabla \gamma_{\delta}\right|+\left|\operatorname{Hess} \gamma_{\delta}\right| \leqslant \delta$ and that the function

$$
H_{1}(x, y)=H(x, y)+\gamma_{\delta}(x, y)=\frac{|x y|^{p}}{p t^{p-1}}-F(u(x), u(y))-v(x)+\gamma_{\delta}(x, y)
$$

has a minimal point $\left(x^{o}, y^{o}\right) \in \mathscr{A}$ with

$$
d_{X \times X}^{2}\left((\bar{x}, \bar{y}),\left(x^{o}, y^{o}\right)\right)=\left|\bar{x} x^{o}\right|^{2}+\left|\bar{y} y^{o}\right|^{2}<\delta^{2} .
$$

Let $\sigma:\left[0,\left|x^{o} y^{o}\right|\right] \rightarrow M$ be a shortest geodesic with $\sigma(0)=x^{o}$ and $\sigma\left(\left|x^{o} y^{o}\right|\right)=y^{o}$ and let $T_{\sigma(t)}: T_{x^{o}} M \rightarrow T_{\sigma(t)} M$ be the parallel transport along $\sigma(t)$. Denote by $T:=T_{y^{o}}$. We want to consider the asymptotic behavior of

$$
\begin{aligned}
I\left(\varepsilon_{j}\right): & =\int_{B_{\varepsilon_{j}}(0) \subset T_{x^{o}} M=\mathbb{R}^{n}}\left[H_{1}\left(\exp _{x^{o}}(\eta), \exp _{y^{o}}(T \eta)\right)-H_{1}\left(x^{o}, y^{o}\right)\right] \mathrm{d} \eta \\
& =I_{1}\left(\varepsilon_{j}\right)-I_{2}\left(\varepsilon_{j}\right)-I_{3}\left(\varepsilon_{j}\right)+I_{4}\left(\varepsilon_{j}\right),
\end{aligned}
$$

where the sequence $\left\{\epsilon_{j}\right\}$ is given in Lemma 3.3 and

$$
\begin{aligned}
& I_{1}\left(\varepsilon_{j}\right):=\frac{1}{p t^{p-1}} \cdot \int_{B_{\varepsilon_{j}}(0)}\left(\left|\exp _{x^{o}}(\eta) \exp _{y^{o}}(T \eta)\right|^{p}-\left|x^{o} y^{o}\right|^{p}\right) \mathrm{d} \eta \\
& I_{2}\left(\varepsilon_{j}\right):=\int_{B_{\varepsilon_{j}}(0)}\left(F\left(\exp _{x^{o}}(\eta), \exp _{y^{o}}(T \eta)\right)-F\left(x^{o}, y^{o}\right)\right) \mathrm{d} \eta \\
& I_{3}\left(\varepsilon_{j}\right):=\int_{B_{\varepsilon_{j}}(0)}\left(v\left(\exp _{x^{o}}(\eta)\right)-v\left(x^{o}\right)\right) d H^{n}(\eta), \\
& \left.I_{4}\left(\varepsilon_{j}\right):=\int_{B_{\varepsilon_{j}}(0)}\left(\gamma_{\delta}\left(\exp _{x^{o}}(\eta), \exp _{y^{o}}(T \eta)\right)\right)-\gamma_{\delta}\left(x^{o}, y^{o}\right)\right) \mathrm{d} \eta
\end{aligned}
$$

The minimal property of point $\left(x^{o}, y^{o}\right)$ implies that

$$
I\left(\varepsilon_{j}\right) \geqslant 0 .
$$

We need to estimate $I_{1}, I_{2}, I_{3}$ and $I_{4}$.

(i) The estimate of $I_{1}$ and $I_{4}$.

Let $T$ be the parallel transportation, the first and the second variation of arc-length implies that

$$
\left|\exp _{x^{o}}(\eta) \exp _{y^{o}}(T \eta)\right|-\left|x^{o} y^{o}\right| \leqslant \frac{\epsilon^{2}}{2} \int_{0}^{\left|x^{o} y^{o}\right|}-R\left(\sigma^{\prime}(t), T_{\gamma(t)} \eta, \sigma^{\prime}(t), T_{\gamma(t)} \eta\right) \mathrm{d} t+o\left(\epsilon^{2}\right)
$$

for all $\eta \in B_{\epsilon}(0)$, where $R(\cdot, \cdot, \cdot, \cdot)$ is the Riemannian curvature tensor. By taking $\epsilon=\varepsilon_{j}$ and using the fact that $a \leqslant b+\delta$ implies $a^{p} \leqslant b^{p}+p \delta b^{p-1}+o(\delta)$ as $\delta \rightarrow 0$, and then integrating over $B_{\varepsilon_{j}}(0)$ 
we obtain

$$
I_{1}\left(\varepsilon_{j}\right) \leqslant \frac{1}{p t^{p-1}} \cdot \frac{p K \cdot \omega_{n-1}}{2 n(n+2)} \cdot\left|x^{o} y^{o}\right|^{p} \cdot \varepsilon_{j}^{n+2}+o\left(\varepsilon_{j}^{n+2}\right)
$$

for any $j \in \mathbb{N}$, where we have used $\operatorname{Ric}\left(\sigma^{\prime}, \sigma^{\prime}\right) \geqslant-K$ and the Fatou's Lemma (since $\left|\exp _{x^{o}}(\eta) \exp _{y^{o}}(T \eta)\right|-$ $\left|x^{o} y^{o}\right| \leqslant C \epsilon^{2}$ for some constant $C$ depending on the sectional curvature on $\left.B_{10\left|x^{o} y^{o}\right|}\left(x^{o}\right)\right)$.

Since $\gamma_{\delta}$ is smooth and that $\mid$ Hess $\gamma_{\delta} \mid \leqslant \delta$, it is easy to check that

$$
I_{4}\left(\varepsilon_{j}\right) \leqslant C(n) \cdot \delta \cdot \varepsilon_{j}^{n+2}+o\left(\varepsilon_{j}^{n+2}\right),
$$

for any $j \in \mathbb{N}$, and for some constant $C(n)>0$.

(ii) The estimate of $I_{2}$.

We put

$$
P=u\left(\exp _{x^{o}}(\eta)\right), \quad Q=u\left(x^{o}\right), \quad W=u\left(y^{o}\right) \quad \text { and } \quad S=u\left(\exp _{y^{o}}(T \eta)\right),
$$

and

$$
l_{0}:=2 \sin \frac{d_{Q W}}{2}, \quad l_{1}:=2 \cos \frac{d_{Q W}}{2}, \quad \alpha=\frac{1}{1+2 l_{0}} \in(0,1) .
$$

Denote the midpoint of $Q, W$ by $Q_{m}$. Note that $\frac{1-\alpha}{2}=\alpha \cdot l_{0}$. Then, by Lemma 2.3 we have that for any $\beta>0$

$$
\begin{aligned}
\alpha l_{0} \cdot( & \left.F\left(x^{o}, y^{o}\right)-F\left(\exp _{x^{o}}(\eta), \exp _{y^{o}}(T \eta)\right)\right) \\
= & \alpha l_{0}\left(\left(2 \sin \frac{d_{Q R}}{2}\right)^{2}-\left(2 \sin \frac{d_{P S}}{2}\right)^{2}\right)+\alpha l_{0}\left(2 \sin \frac{d_{Q R}}{2}-2 \sin \frac{d_{P S}}{2}\right) \\
\leqslant & {\left[1-\frac{1-\alpha}{2}\left(1-\frac{1}{\beta}\right)\right]\left(2 \sin \frac{d_{P Q}}{2}\right)^{2}+l_{1} \cdot\left(\cos d_{P Q_{m}}-\cos d_{Q Q_{m}}\right) } \\
& +\left[1-\frac{1-\alpha}{2}(1-\beta)\right]\left(2 \sin \frac{d_{R S}}{2}\right)^{2}+l_{1} \cdot\left(\cos d_{S Q_{m}}-\cos d_{R Q_{m}}\right) \\
\leqslant & {\left[w_{a_{1}, b, Q_{m}, x^{o}}\left(\exp _{x^{o}}(\eta)\right)-w_{a_{1}, b, Q_{m}, x^{o}}\left(x^{o}\right)\right] } \\
& +\left[w_{a_{2}, b, Q_{m}, y^{o}}\left(\exp _{y^{o}}(T \eta)\right)-w_{a_{2}, b, Q_{m}, y^{o}}\left(y^{o}\right)\right],
\end{aligned}
$$

where the function $w_{a, b, Q_{m}, x^{o}}$ is given in Lemma 3.3 with the constants

$$
a_{1}:=1-\frac{1-\alpha}{2}\left(1-\frac{1}{\beta}\right), \quad b:=l_{1}, \quad a_{2}:=1-\frac{1-\alpha}{2}(1-\beta),
$$

and we have used $2 \sin (t / 2) \leqslant t$ for any $t \in(0, \pi)$. From $\rho_{0}=\operatorname{diam}\left(u\left(B_{2 R}\right)\right)$ we have $u\left(B_{2 R}\right) \subset$ $\overline{B_{\rho_{0}}\left(u\left(x_{0}\right)\right)} \cap \overline{B_{\rho_{0}}\left(u\left(y_{0}\right)\right)}$. By the assumption $\rho_{0}<\pi / 2$ and that $X$ is a $C A T(1)$-space, we obtain $u\left(B_{2 R}\right) \subset \overline{B_{\rho_{0}}\left(Q_{m}\right)}$. Integrating over $B_{\varepsilon_{j}}\left(x^{o}\right)$, Lemma 3.3 implies that

$$
\begin{aligned}
& \alpha l_{0} \cdot \int_{B_{\varepsilon_{j}}\left(x^{o}\right)}\left(F\left(x^{o}, y^{o}\right)-F\left(\exp _{x^{o}}(\eta), \exp _{y^{o}}(T \eta)\right)\right) \mathrm{d} \eta \\
& \leqslant {\left[2 a_{1}-b \cdot \cos \left(d_{X}\left(u\left(x^{o}\right), Q_{m}\right)\right)\right] \cdot \frac{\omega_{n-1} \cdot e_{u}\left(x^{o}\right)}{2 n(n+2)} \cdot \epsilon_{j}^{n+2}+} \\
&+\left[2 a_{2}-b \cdot \cos \left(d_{X}\left(u\left(y^{o}\right), Q_{m}\right)\right)\right] \cdot \frac{\omega_{n-1} \cdot e_{u}\left(y^{o}\right)}{2 n(n+2)} \cdot \epsilon_{j}^{n+2}+o\left(\epsilon_{j}^{n+2}\right) .
\end{aligned}
$$

Noticing that $\cos d_{X}\left(Q_{m}, u\left(x^{o}\right)\right)=l_{1} / 2$ and that $1-l_{1}^{2} / 4=l_{0}^{2} / 4$, we choose $\beta$ such that

$$
a_{2}=\frac{l_{1}^{2}}{4} \quad\left(\Longleftrightarrow \beta=1-\frac{l_{0}\left(1+2 l_{0}\right)}{4}\right),
$$


where we have used $\alpha=1 /\left(1+2 l_{0}\right)$. Notice that $\beta>0$ provided $l_{0} \leqslant 1$. Then we have

$$
\begin{aligned}
-I_{2}\left(\varepsilon_{j}\right) & =\int_{B_{\varepsilon_{j}}(0)}\left(F\left(x^{o}, y^{o}\right)-F\left(\exp _{x^{o}}(\eta), \exp _{y^{o}}(T \eta)\right)\right) \mathrm{d} \eta \\
& \leqslant \frac{a_{1}-l_{1}^{2} / 4}{\alpha l_{0}} \cdot \frac{\omega_{n-1} e_{u}\left(x^{o}\right)}{n(n+2)} \cdot \epsilon_{j}^{n+2}++o\left(\varepsilon_{j}^{n+2}\right) .
\end{aligned}
$$

From $1-\beta=\frac{l_{0}}{4 \alpha}$ and $\frac{1}{\alpha}=1+2 l_{0}$, we have, if $l_{0} \leqslant \varepsilon / 6$, that

$$
\begin{aligned}
\frac{a_{1}-l_{1}^{2} / 4}{\alpha l_{0}} & =\frac{1-\frac{1-\alpha}{2}\left(1-\frac{1}{\beta}\right)-l_{1}^{2} / 4}{\alpha l_{0}} \\
& =l_{0}\left(1+2 l_{0}\right)\left(\frac{1}{4}+\frac{1}{4-l_{0}\left(1+2 l_{0}\right)}\right) \\
& \leqslant \frac{l_{0}}{2}(1+\varepsilon) .
\end{aligned}
$$

When both $t$ and $\delta$ are small enough, the combination of Eq.4.6) and Lemma4.1(3) implies that $l_{0} \leqslant \varepsilon / 6$. Therefore, we by 4.15] get that

$$
-I_{2}\left(\varepsilon_{j}\right) \leqslant(1+\varepsilon) \frac{l_{0}}{2} \cdot \frac{\omega_{n-1}}{n(n+2)} e_{u}\left(x^{o}\right) \cdot \varepsilon_{j}^{n+2}+o\left(\varepsilon_{j}^{n+2}\right) .
$$

(iii) The estimate of $I_{3}$.

By Corollary 3.2 and the definition of $v$, we have

$$
-I_{3}\left(\varepsilon_{j}\right) \leqslant\left(\frac{-K}{t^{p-1}} \cdot L_{t}^{p}\left(x^{o}\right)-(1+\varepsilon) e_{u}\left(x^{o}\right) D_{t}\left(x^{o}\right)-\theta_{0}\right) \cdot \frac{\omega_{n-1}}{2 n(n+2)} \cdot \varepsilon_{j}^{n+2}+o\left(\varepsilon_{j}^{n+2}\right) .
$$

By combining these estimates for $I_{1}, I_{2}, I_{3}$ and $I_{4}$, we have

$$
\begin{aligned}
& \frac{K}{t^{p-1}}\left(\left|x^{o} y^{o}\right|^{p}-L_{t}^{p}\left(x^{o}\right)\right)+(1+\varepsilon) e_{u}\left(x^{o}\right)\left(2 \sin \frac{d_{X}\left(u\left(x^{o}\right), u\left(y^{o}\right)\right)}{2}-D_{t}\left(x^{o}\right)\right) \\
& -\theta_{0}+C(n) \frac{2 n(n+2)}{\omega_{n-1}} \delta \geqslant 0 .
\end{aligned}
$$

Eq.(4.6) implies that $\left(x^{o}, y^{o}\right)$ converge to $(\bar{x}, \bar{y})$ as $\delta \rightarrow 0$. We by the lower semi-continuity of $L_{t}$ and $D_{t}$ have that

and

$$
\liminf _{\delta \rightarrow 0} L_{t}\left(x^{o}\right) \geqslant L_{t}(\bar{x})=\lim _{\delta \rightarrow 0}\left|x^{o} y^{o}\right|
$$

$$
\liminf _{\delta \rightarrow 0} D_{t}\left(x^{o}\right) \geqslant D_{t}(\bar{x})=F(\bar{x}, \bar{y})=\lim _{\delta \rightarrow 0} 2 \sin \frac{d_{X}\left(u\left(x^{o}\right), u\left(y^{o}\right)\right)}{2} .
$$

A contradiction appears in (4.16) when we take $\delta \rightarrow 0$ (noticing that $K \geqslant 0$ ). The proof is finished.

\subsection{The Bochner inequality.}

We will prove Theorem 1.10 in this subsection.

Let $p \in(1, \infty)$ and let $f_{t}$ be the auxiliary functions defined as in (4.1) in the previous subsection, on a ball $B_{R}$ with $B_{2 R} \subset \subset \Omega$.

Lemma 4.4. (i) Let $q \in(1, \infty)$ with $1 / q+1 / p=1$. For any $x \in B_{R}$, we have

$$
\liminf _{t \rightarrow 0} \frac{f_{t}(x)}{t} \geqslant-\frac{1}{q} \operatorname{Lip}^{q} u(x)
$$


(ii) If $u$ is metrically differentiable at $x$, then we have

$$
\lim _{t \rightarrow 0^{+}} \frac{f_{t}(x)}{t}=-\frac{G_{u}^{q}(x)}{q}
$$

and

$$
\lim _{t \rightarrow 0^{+}} \frac{L_{t}(x)}{t}=G_{u}^{q / p}(x), \quad \lim _{t \rightarrow 0^{+}} \frac{D_{t}(x)}{t}=G_{u}^{q}(x) .
$$

Proof. (i) By the basic inequality $a^{p} / p-a b \geqslant-b^{q} / q$ for any $a, b \in \mathbb{R}$, we have

$$
\frac{1}{p} \cdot \frac{|x y|^{p}}{t^{p}}-\frac{F(x, y)}{t} \geqslant-\frac{1}{q}\left(\frac{F(x, y)}{|x y|}\right)^{q}, \quad \forall x, y \in B_{2 R} .
$$

Taking $y_{t} \in S_{t}(x)$ with $\left|x y_{t}\right|=L_{t}(x)$, we obtain from the definition of $f_{t}$ that

$$
\begin{aligned}
\liminf _{t \rightarrow 0} \frac{f_{t}}{t} \geqslant & -\frac{1}{q} \limsup _{y_{t} \rightarrow x}\left(\frac{F\left(x, y_{t}\right)}{\left|x y_{t}\right|}\right)^{q}=-\frac{1}{q} \limsup _{t \rightarrow 0}\left(\frac{D_{t}(x)}{L_{t}(x)}\right)^{q} \\
& \geqslant-\frac{1}{q} \operatorname{Lip}^{q} u(x),
\end{aligned}
$$

where we have used $\lim _{y \rightarrow x} F(x, y) / d_{X}(u(x), u(y))=1$. This proves (4.17).

(ii) Let $u$ be metrically differentiable at $x$. Take a unit vector $\xi \in T_{x} M$ such that

$$
G_{u}(x)=m d u_{x}(\xi) .
$$

For each small $t>0$, we put $y_{t, x}:=\exp _{x}\left(t G_{u}^{q / p} \cdot \xi\right)$. Then

$$
\left|x y_{t, x}\right|=t \cdot G_{u}^{q / p}(x)
$$

and

$$
\begin{aligned}
d_{X}\left(u(x), u\left(y_{t, x}\right)\right) & =\left|x y_{t, x}\right| \cdot m d u_{x}(\xi)+o\left(\left|x y_{t, x}\right|\right) \\
& =t \cdot G_{u}^{q / p+1}(x)+o(t)=t \cdot G_{u}^{q}(x)+o(t),
\end{aligned}
$$

as $t \rightarrow 0$. Thus, by the definition of $f_{t}$, we obtain

$$
\frac{f_{t}(x)}{t} \leqslant \frac{\left|x y_{t, x}\right|^{p}}{p t^{p}}-\frac{F\left(x, y_{t, x}\right)}{t}=\frac{G_{u}^{q}(x)}{p}-G_{u}^{q}(x)+o(1)=-\frac{G_{u}^{q}(x)}{q}+o(1) .
$$

as $t \rightarrow 0$. That is

$$
\limsup _{t \rightarrow 0^{+}} \frac{f_{t}(x)}{t} \leqslant-\frac{G_{u}^{q}(x)}{q}
$$

Recall that $G_{u}(x)=\operatorname{Lip} u(x)$ by Lemma2.9, the combination of (4.17) and (4.21) yields (4.18).

Combining (4.18) and (4.20) together gives

$$
\limsup _{t \rightarrow 0}\left(\frac{D_{t}(x)}{L_{t}(x)}\right)^{q}=\operatorname{Lip}^{q} u(x) .
$$

On the other hand, notice that

$$
-\frac{f_{t}(x)}{t}=-\frac{L_{t}^{p}(x)}{p t^{p}}+\frac{D_{t}(x)}{L_{t}(x)} \cdot \frac{L_{t}(x)}{t} \leqslant \frac{1}{q}\left(\frac{D_{t}(x)}{L_{t}(x)}\right)^{q} .
$$

Thus, we get

Therefore, we obtain

$$
\liminf _{t \rightarrow 0} \frac{D_{t}(x)}{L_{t}(x)} \geqslant \operatorname{Lip} u(x)
$$

$$
\lim _{t \rightarrow 0} \frac{D_{t}(x)}{L_{t}(x)}=\operatorname{Lip} u(x) .
$$


By using $f_{t} / t=\frac{L_{t}^{p}}{p t^{p}}-\frac{D_{t}}{L_{t}} \cdot \frac{L_{t}}{t}$ again, it follows $\lim _{t \rightarrow 0} \frac{L_{t}(x)}{t}=\operatorname{Lip}^{\frac{1}{p-1}} u(x)=\operatorname{Lip}^{q / p} u(x)$, and then $\lim _{t \rightarrow 0} \frac{D_{t}(x)}{t}=\operatorname{Lip}^{1+q / p} u(x)=\operatorname{Lip}^{q} u(x)$. The proof is finished.

Now we are in the place to prove Theorem 1.10 .

Proof of Theorem 1.10. We have known that $\operatorname{Lip} \in L_{\text {loc }}^{\infty}(\Omega)$ from Theorem 1.3. By a rescaling argument of the target space, we can assume that $\left(X, d_{X}\right)$ is a $C A T(1)$-space. In this case, since Lip $u=G_{u}$ for almost all $x \in \Omega$, we need to prove $G_{u} \in W_{\text {loc }}^{1,2}(\Omega)$ and that

$$
\Delta G_{u} \geqslant-K G_{u}-e_{u} \cdot G_{u}
$$

on $\Omega$, in the sense of distributions. It suffices to show that: for any $o \in \Omega$, there exists a neighborhood $B_{R}(o)$ with $B_{2 R}(o) \subset \Omega$ such that $G_{u} \in W_{\text {loc }}^{1,2}\left(B_{R}(o)\right)$ and that (4.22) holds on $B_{R}(o)$, in the sense of distributions.

Since $u$ is continuous (from Theorem 2.4), for any $o \in \Omega$, there exists a neighborhood $B_{R}(o)$ such that Image $\left(u\left(B_{2 R}(o)\right)\right) \subset B_{\pi / 4}(u(o))$. In particular, the triangle inequality yields $\operatorname{diam} u\left(B_{2 R}(o)\right)<\pi / 2$. Fix such a neighborhood $B_{R}=B_{R}(o)$.

Fix any $p \in[2, \infty)$. From Lemma 4.1 (3), we get $\Delta f_{t} / t \leqslant C_{\ell_{0}}$ on $B_{3 R / 2}$ for any $t \in\left(0, t_{*}\right)$, where the constant $C_{\ell_{0}}$ is uniformly with respect to $t \in\left(0, t_{*}\right)$. By combining Lemma 4.4 and Proposition 4.2 together, we have, for any $\varepsilon>0$ that $G_{u}^{q} / q \in W_{\mathrm{loc}}^{1,2}\left(B_{3 R / 2}\right)$ and that

$$
\Delta\left(G_{u}^{q} / q\right) \geqslant-K G_{u}^{q}-(1+\varepsilon) \cdot e_{u} \cdot G_{u}^{q}
$$

on $B_{3 R / 2}$, in the sense of distributions, where $q=p /(p-1) \in(1,2]$. From the arbitrariness of $\varepsilon$, we conclude that

$$
\Delta\left(G_{u}^{q} / q\right) \geqslant-K G_{u}^{q}-e_{u} \cdot G_{u}^{q}
$$

on $B_{3 R / 2}$, in the sense of distributions, where $q=p /(p-1) \in(1,2]$.

In order to take the limit of (4.23) as $q \rightarrow 1$, we want to show that the energies $\left\|\nabla\left(G_{u}^{q} / q\right)\right\|_{L^{2}\left(B_{R}\right)}$ are bounded uniformly with respect to $q$. By the local Lipschitz continuity of $u$, there exists a constant $C_{1}>1$ such that $G_{u}, e_{u} \leqslant C_{1}$ on $B_{3 R / 2}$. Hence, we have

$$
\Delta\left(G_{u}^{q} / q\right) \geqslant-K \cdot C_{1}^{q}-C_{1}^{q+1} \geqslant-K \cdot C_{1}^{2}-C_{1}^{3}
$$

on $B_{3 R / 2}$, in the sense of distributions, where we have used $K \geqslant 0$ and $q \in(1,2]$. By applying Caccioppoli inequality, we conclude that the energies $\left\|\nabla\left(G_{u}^{q} / q\right)\right\|_{L^{2}\left(B_{R}\right)}$ are bounded uniformly. Hence, there exists a sequence $\left\{q_{j}\right\}_{j \in \mathbb{N}}$ with $q_{j} \in(1,2]$ and $q_{j} \searrow 1$ such that $\Delta\left(G_{u}^{q_{j}} / q_{j}\right) \rightarrow \Delta G_{u}$. Now, by letting $q_{j} \rightarrow 1$ in (4.23), we conclude that $G_{u} \in W^{1,2}\left(B_{R}\right)$ and 4.22). The proof is finished.

\section{YAU'S GRADIENT ESTIMATES}

We will continue to assume that $\Omega$ is a smooth domain of an $n$-dimensional Riemannian manifold $(M, g)$ with Ric $\geqslant-K$ for some $K \geqslant 0$, and that $\left(X, d_{X}\right)$ is a $C A T(\kappa)$-space for some $\kappa \geqslant 0$. Let $u$ be a harmonic map from $\Omega$ to $X$. Assume that its image $u(\Omega) \subset X$ is contained in a ball with radius $<\frac{\pi}{2 \sqrt{\kappa}}$.

When the target space has non-positive curvature, we have the following a consequence of the Bochner inequality, Theorem 1.10 .

Lemma 5.1. Let $\kappa=0$. Suppose that $B_{2 R}\left(x_{0}\right) \subset \subset \Omega$ and that $u\left(B_{R}\left(x_{0}\right)\right) \subset B_{\rho}\left(Q_{0}\right)$ for some $\rho>0$ and $Q_{0} \in X$. We put

$$
h=2 \rho^{2}-d_{X}^{2}\left(Q_{0}, u(x)\right) \text { and } F=\frac{\operatorname{Lip} u}{h} .
$$


Then $F \in W^{1,2} \cap L^{\infty}\left(B_{R}\left(x_{0}\right)\right)$ and

$$
\Delta F+2\langle\nabla F, \nabla \log h\rangle \geqslant C \rho^{2} \cdot F^{3}-K \cdot F
$$

in the sense of distribution, where $C=C_{n, \sqrt{K} R}$.

Proof. From Theorem 1.10, we have Lip $u \in W^{1,2} \cap L^{\infty}\left(B_{R}\left(x_{0}\right)\right)$. Noticing that $h$ is Lipschitz continuous and $h \geqslant \rho^{2}$, we obtain $F \in W^{1,2} \cap L^{\infty}\left(B_{R}\left(x_{0}\right)\right)$.

By applying the Chain rule to Lip $u=h F$, we have

$$
h \cdot \Delta F+2\langle\nabla F, \nabla h\rangle+F \cdot \Delta h=\Delta \operatorname{Lip} u .
$$

Multiplying both sides of this inequality by $h^{-1}$ and substituting (1.7) and then $-\Delta h \geqslant 2 e_{u}$ (see Lemma 2.5), we get

$$
\Delta F+2\langle\nabla F, \nabla \log h\rangle=-F \cdot \frac{\Delta h}{h}+\frac{\Delta \operatorname{Lip} u}{h} \geqslant F \cdot \frac{2 e_{u}}{h}-K F .
$$

in the sense of distributions. As Corollary 2.6, we have

$$
\begin{aligned}
\Delta F+2\langle\nabla F, \nabla \log h\rangle & \geqslant C_{n, \sqrt{K} R} \frac{F}{h} \cdot \operatorname{Lip}^{2} u-K F=C_{n, \sqrt{K} R} h F^{3}-K F \\
& \geqslant C_{n, \sqrt{K} R} \cdot \rho^{2} \cdot F^{3}-K F
\end{aligned}
$$

where we have used $h \geqslant \rho^{2}$ again. The proof is finished.

Similarly, in the case of where the target is a $C A T(1)$-space, we have the following property.

Lemma 5.2. Let $\kappa=1$. Suppose that $B_{2 R}\left(x_{0}\right) \subset \subset \Omega$ and that $u\left(B_{R}\left(x_{0}\right)\right) \subset B_{\rho}\left(Q_{0}\right)$ for some $\rho<\pi / 2$ and $Q_{0} \in X$. We put $\rho_{0}=\frac{\rho+\pi / 2}{2}=\rho+\frac{\pi / 2-\rho}{2}$ and

$$
h_{1}=\cos d_{X}\left(Q_{0}, u(x)\right)-\cos \rho_{0} \quad \text { and } \quad F=\frac{\operatorname{Lip} u}{h_{1}} .
$$

Then $F \in W^{1,2} \cap L^{\infty}\left(B_{R}\left(x_{0}\right)\right)$ and

$$
\Delta F+2\left\langle\nabla F, \nabla \log h_{1}\right\rangle \geqslant C^{\prime} \cdot F^{3}-K F
$$

in the sense of distribution, where $C^{\prime}=C^{\prime}(n, \sqrt{K} R, \pi / 2-\rho)$ is a constant depends on $n, \sqrt{K} R$ and $\pi / 2-\rho$.

Proof. It is easy to see that $h_{1}$ is Lipschitz continuous and

$$
h_{1} \geqslant \cos \rho-\cos \rho_{0}:=C_{1}^{\prime}>0
$$

for some positive number $C_{1}^{\prime}$ depends only on $\pi / 2-\rho$. From Lemma 2.5, we have

$$
-\Delta h_{1} \geqslant \cos d_{X}^{2}\left(Q_{0}, u(x)\right) \cdot e_{u} \geqslant \cos \rho \cdot e_{u} .
$$

The remain of the proof, by a similar argument as in Lemma5.1, we have

$$
\Delta F+2\left\langle\nabla F, \nabla \log h_{1}\right\rangle \geqslant C_{n, \sqrt{K} R} \cdot C_{1}^{\prime} \cdot \cos \rho \cdot F^{3}-K F
$$

in the sense of distributions.

In general, the function Lip $u$ does not smooth, even may not be continuous. It is difficult to employ the argument in [4] directly. So we will ues the following the approximating version of the maximum principle. 
Theorem 5.3. ([46, Theorem 1.4]) Let $f(x) \in W_{\mathrm{loc}}^{1,2} \cap L_{\mathrm{loc}}^{\infty}(\Omega)$ such that $\Delta f$ is a signed Radon measure with $\Delta^{\text {sing }} f \geqslant 0$, where $\Delta f=\Delta^{\mathrm{ac}} f \cdot \operatorname{vol}_{g}+\Delta^{\operatorname{sing}} f$ is the Radon-Nikodym decomposition with respect to $\operatorname{vol}_{g}$. Suppose that $f$ achieves one of its strict maximum in $\Omega$ in the sense that: there exists a neighborhood $U \subset \subset \Omega$ such that

$$
\sup _{U} f>\sup _{\Omega \backslash U} f .
$$

Then, given any $w \in W^{1,2}(\Omega) \cap L^{\infty}(\Omega)$, there exists a sequence of points $\left\{x_{j}\right\}_{j \in \mathbb{N}} \subset U$ such that they are the approximate continuity points of $\Delta^{\mathrm{ac}} f$ and $\langle\nabla f, \nabla w\rangle$, and that

$$
f\left(x_{j}\right) \geqslant \sup _{\Omega} f-1 / j \quad \text { and } \quad \Delta^{\mathrm{ac}} f\left(x_{j}\right)+\langle\nabla f, \nabla w\rangle\left(x_{j}\right) \leqslant 1 / j .
$$

Here and in the sequel, $\sup _{U} f$ means $\operatorname{ess}-\sup _{U} f$.

Proof. It was proved in [46] in the setting of metric measure spaces with generalized Ricci curvature bounded from below. In particular, it holds for Riemannian manifolds with Ricci curvature bounded from below (with the Riemannian measure).

The proof of the Theorem 1.4 and Theorem 1.7 are both based on the following lemma.

Lemma 5.4. Let $B_{R}\left(x_{0}\right) \subset \Omega$ and let $F \in W_{\mathrm{loc}}^{1,2} \cap L_{\mathrm{loc}}^{\infty}\left(B_{R}\left(x_{0}\right)\right)$ be a nonnegative function. Assume that $F$ satisfies

$$
\Delta F+\langle\nabla F, \nabla v\rangle \geqslant a_{1} F^{3}-a_{2} F,
$$

in the sense of distributions, where $v \in W_{\mathrm{loc}}^{1,2} \cap L_{\mathrm{loc}}^{\infty}\left(B_{R}\left(x_{0}\right)\right)$ such that $|\nabla v| \leqslant a_{3} F$, and the constants $a_{1}, a_{3}>0$ and $a_{2} \geqslant 0$. Then there exists a constant $C_{n, \sqrt{K} R}$ such that

$$
\sup _{B_{R / 2}\left(x_{0}\right)} F^{2} \leqslant \frac{2 a_{2}}{a_{1}}+\frac{C_{n, \sqrt{K} R}}{R^{2}}\left(\frac{1}{a_{1}}+\frac{a_{3}^{2}}{a_{1}^{2}}\right) .
$$

Proof. Fix any a small number $\delta$ such that $0<\delta<\frac{1}{2} \frac{\sup _{B_{R / 2} F} F}{\sup _{B_{3 R / 4}} F}$. Let us choose $\eta(x)=\eta(r(x))$ to be a function of the distance $r$ to the fixed point $x_{0}$ with the following property that

$$
\delta \leqslant \eta \leqslant 1 \text { on } B_{R}, \quad \eta=1 \text { on } B_{R / 2}, \quad \eta=\delta \text { on } B_{R} \backslash B_{3 R / 4},
$$

and

$$
-\frac{C_{1}}{R} \leqslant \eta^{\prime}(r) \leqslant 0 \quad \text { and } \quad\left|\eta^{\prime \prime}(r)\right| \leqslant \frac{C_{1}}{R^{2}} \quad \forall r \in(0,3 R / 4)
$$

for some universal constant $C_{1}$ (which is independent of $n, K, R$ ). Then we have

$$
|\nabla \eta|=\left|\eta^{\prime}\right||\nabla r| \leqslant \frac{C_{1}}{R} \quad \text { on } B_{3 R / 4},
$$

and, by the Laplacian comparison theorem, that

$$
\begin{aligned}
\Delta \eta & =\eta^{\prime} \Delta r+\eta^{\prime \prime}|\nabla r|^{2} \geqslant-\frac{C_{1}}{R}(\sqrt{(n-1) K} \operatorname{coth}(r \sqrt{K /(n-1)}))-\frac{C_{1}}{R^{2}} \\
& \geqslant-\frac{C_{1}}{R}\left(\sqrt{(n-1) K}+\frac{n-1}{R}\right)-\frac{C_{1}}{R^{2}}=-\frac{C_{1} \sqrt{(n-1) K} R+n C_{1}}{R^{2}} \geqslant-\frac{C_{2}}{R^{2}}
\end{aligned}
$$

on $B_{3 R / 4}$, in the sense of distributions, where we have used that

$$
\operatorname{coth}(r \sqrt{K /(n-1)}) \leqslant \operatorname{coth}(R \sqrt{K /(n-1)}) \leqslant 1+\frac{1}{R \sqrt{K /(n-1)}} .
$$

Here and in the sequel of this proof, we denote $C_{1}, C_{2}, C_{3}, \cdots$ the various constants which depend only on $n$ and $\sqrt{K} R$. 
Now we put $G=\eta F$. Then $G$ is in $W^{1,2}\left(B_{3 R / 4}\right) \cap L^{\infty}\left(B_{3 R / 4}\right)$ and $G$ achieves one of its strict maximum in $B_{R / 2}$ in the sense of Theorem 5.3 ,

$$
\begin{aligned}
\Delta G+\langle\nabla G, \nabla v\rangle & =\Delta \eta \cdot F+2\langle\nabla \eta, \nabla(G / \eta)\rangle+\eta \cdot \Delta F+\eta\langle\nabla F, \nabla v\rangle+F\langle\nabla \eta, \nabla v\rangle \\
& \geqslant \Delta \eta \cdot \frac{G}{\eta}+2\langle\nabla \log \eta, \nabla G\rangle-2 \frac{|\nabla \eta|^{2}}{\eta} \cdot \frac{G}{\eta}+\eta(\Delta F+\langle\nabla F, \nabla v\rangle)+\frac{G}{\eta}\langle\nabla \eta, \nabla v\rangle \\
& \geqslant-\frac{G}{\eta} \frac{C_{2}}{R^{2}}+2\langle\nabla \log \eta, \nabla G\rangle-\frac{G}{\eta^{2}} \frac{2 C_{1}^{2}}{R^{2}}+\eta \cdot\left(a_{1} F^{3}-a_{2} F\right)+\frac{G}{\eta}\langle\nabla \eta, \nabla v\rangle .
\end{aligned}
$$

By setting $w=v-2 \log \eta \in W^{1,2}\left(B_{3 R / 4}\right) \cap L^{\infty}\left(B_{3 R / 4}\right)$ and using $|\nabla v| \leqslant a_{3} F=a_{3} \frac{G}{\eta}$, we have

$$
\begin{aligned}
\Delta G+\langle\nabla G, \nabla w\rangle & \geqslant-\frac{C_{2}}{R^{2}} \frac{G}{\eta}-\frac{2 C_{1}^{2}}{R^{2}} \frac{G}{\eta^{2}}+\eta\left(a_{1}(G / \eta)^{3}-a_{2}(G / \eta)\right)-a_{3} \frac{G}{\eta}|\nabla \eta| \frac{G}{\eta} \\
& \geqslant-\frac{C_{2}}{R^{2}} \frac{G}{\eta}-\frac{2 C_{1}^{2}}{R^{2}} \frac{G}{\eta^{2}}+a_{1} \frac{G^{3}}{\eta^{2}}-a_{2} G-a_{3} \frac{G^{2}}{\eta^{2}} \frac{C_{1}}{R} \\
& \geqslant-\frac{C_{2}}{R^{2}} \frac{G}{\eta} \frac{1}{\eta}-\frac{2 C_{1}^{2}}{R^{2}} \frac{G}{\eta^{2}}+a_{1} \frac{G^{3}}{\eta^{2}}-a_{2} G \frac{1}{\eta^{2}}-a_{3} \frac{G^{2}}{\eta^{2}} \frac{C_{1}}{R} \\
& \geqslant \frac{G}{\eta^{2}}\left[-\frac{C_{2}}{R^{2}}-\frac{2 C_{1}^{2}}{R^{2}}+a_{1} G^{2}-a_{2}-a_{3} \frac{C_{1}}{R} G\right],
\end{aligned}
$$

where we have used $G \geqslant 0,1 / \eta \geqslant 1$ and $a_{2} \geqslant 0$. Let $C_{3}:=C_{2}+2 C_{1}^{2}$. Substituting $\frac{a_{3} C_{1}}{R} \cdot G \leqslant$ $\frac{a_{1}}{2} G^{2}+\frac{1}{2 a_{1}}\left(\frac{a_{3} C_{1}}{R}\right)^{2}$ into the above inequality, we obtain

$$
\Delta G+\langle\nabla G, \nabla w\rangle \geqslant \frac{G}{\eta^{2}}\left[-\frac{C_{3}}{R^{2}}+\frac{a_{1}}{2} G^{2}-a_{2}-\frac{C_{1}^{2} a_{3}^{2}}{2 a_{1} R^{2}}\right]
$$

in the sense of distributions. According to Theorem 5.3, there exit a sequence $\left\{x_{j}\right\}_{j \in \mathbb{N}}$ such that, for each $j \in \mathbb{N}$,

$$
G_{j}:=G\left(x_{j}\right) \geqslant \sup _{B_{3 R / 2}} G-1 / j
$$

and that

$$
\frac{G_{j}}{\eta^{2}\left(x_{j}\right)}\left[\frac{a_{1}}{2} G_{j}^{2}-a_{2}-\frac{C_{3}}{R^{2}}-\frac{C_{1}^{2} a_{3}^{2}}{2 a_{1} R^{2}}\right] \leqslant \frac{1}{j} .
$$

As $\eta \geqslant \delta>0$, by letting $j \rightarrow \infty$, we have

$$
\sup _{B_{3 R / 4}} G^{2}=\lim _{j \rightarrow \infty} G_{j}^{2} \leqslant \frac{2 a_{2}}{a_{1}}+\frac{2 C_{3}}{a_{1} R^{2}}+\frac{C_{1}^{2} a_{3}^{2}}{a_{1}^{2} R^{2}} .
$$

This yields

$$
\sup _{B_{R / 2}} F^{2} \leqslant \sup _{B_{3 R / 4}} G^{2} \leqslant \frac{2 a_{2}}{a_{1}}+\frac{2 C_{3}}{a_{1} R^{2}}+\frac{C_{1}^{2} a_{3}^{2}}{a_{1}^{2} R^{2}} \leqslant \frac{2 a_{2}}{a_{1}}+\frac{C_{4}}{R^{2}}\left(\frac{1}{a_{1}}+\frac{a_{3}^{2}}{a_{1}^{2}}\right),
$$

where $C_{4}:=\max \left\{2 C_{3}, C_{1}^{2}\right\}$. The proof is finished.

Now we are in the place to show the main results.

Proof of Theorem 1.4 By applying Lemma 5.4 to (5.1) in Lemma 5.1 with $v=2 \log h$ and

$$
a_{1}=C_{5} \rho^{2}, \quad a_{2}=K, \quad a_{3}=2 \rho,
$$


and noticing that

$$
|\nabla v|=2 \frac{|\nabla h|}{h}=2 \frac{d_{X}\left(Q_{0}, u(x)\right) \cdot\left|\nabla d_{X}\left(Q_{0}, u(x)\right)\right|}{h} \leqslant 2 \frac{\rho \cdot \operatorname{Lip} u}{h}=2 \rho F,
$$

We conclude that, for some constants $C_{5}, C_{6}, C_{7}$ depending only on $n$ and $\sqrt{K} R$, it holds

$$
\sup _{B_{R / 2}\left(x_{0}\right)} \frac{\operatorname{Lip}^{2} u}{\left(2 \rho^{2}-d_{X}^{2}\left(Q_{0}, u(x)\right)\right)^{2}} \leqslant \frac{2 K}{C_{5} \rho^{2}}+\frac{C_{6}}{R^{2}}\left(\frac{1}{C_{5} \rho^{2}}+\frac{4 \rho^{2}}{C_{5}^{2} \rho^{4}}\right) \leqslant \frac{C_{7}}{\rho^{2}}\left(K+\frac{1}{R^{2}}\right) .
$$

This implies

$$
\begin{aligned}
\sup _{B_{R / 2}\left(x_{0}\right)} \operatorname{Lip}^{2} u & \leqslant \frac{C_{7}}{\rho^{2}}\left(K+\frac{1}{R^{2}}\right) \cdot \sup _{B_{R / 2}\left(x_{0}\right)}\left(2 \rho^{2}-d_{X}^{2}\left(Q_{0}, u(x)\right)\right)^{2} \\
& \leqslant C_{7} \frac{4 \rho^{2}}{R^{2}}\left(K R^{2}+1\right)=C_{8} \cdot \frac{\rho^{2}}{R^{2}} .
\end{aligned}
$$

for some constant $C_{8}$ depending only on $n$ and $\sqrt{K} R$. The proof is finished.

Proof of Theorem 1.7 By applying Lemma 5.4 to Lemma 5.2 with $v=2 \log h$ and noticing that

$$
|\nabla v|=2 \frac{|\nabla h|}{h}=2 \frac{\sin d_{X}\left(Q_{0}, u(x)\right) \cdot\left|\nabla d_{X}\left(Q_{0}, u(x)\right)\right|}{h} \leqslant 2 \frac{\operatorname{Lip} u}{h}=2 F,
$$

and choosing

$$
a_{1}=C_{1}^{\prime}, \quad a_{2}=K, \quad a_{3}=2,
$$

Here and in the sequel of this proof, we denote $C_{1}^{\prime}, C_{2}^{\prime}, C_{3}^{\prime}, \cdots$ the various constants which depend only on $n, \sqrt{K} R$ and $\pi / 2-\rho$. We conclude that

$$
\sup _{B_{R / 2}\left(x_{0}\right)} \frac{\operatorname{Lip}^{2} u}{\left(\cos d_{X}\left(Q_{0}, u\right)-\cos \rho_{0}\right)^{2}} \leqslant \frac{2 K}{C_{1}^{\prime}}+\frac{C_{2}^{\prime}}{R^{2}}\left(\frac{1}{C_{1}^{\prime}}+\frac{4}{\left(C_{1}^{\prime}\right)^{2}}\right) \leqslant C_{3}^{\prime}\left(K+\frac{1}{R^{2}}\right),
$$

where $\rho_{0}=\pi / 4+\rho / 2$. By noticing that $\cos \left(d_{X} \circ u\right)-\cos \rho_{0} \leqslant \cos \rho$, this implies

$$
\sup _{B_{R / 2}\left(x_{0}\right)} \operatorname{Lip}^{2} u \leqslant C_{3}^{\prime} \cos \rho\left(K+\frac{1}{R^{2}}\right) \leqslant \frac{C_{4}^{\prime}}{R^{2}} .
$$

The proof is finished.

\section{Appendix A. An generalized Jensen's lemma AND the Proof of Lemma 4.3}

We need a simple lemma for symmetric matrices as follows.

Lemma A.1. Let $A=\left(a_{i j}\right)_{n \times n}, B=\left(b_{i j}\right)_{n \times n}$ be two symmetric matrices. Assume that $B$ is nonnegative definite, and that $\left|a_{i j}-\delta_{i j}\right| \leqslant \frac{1}{2 n^{2}}$ for any $1 \leqslant i, j \leqslant n$, where $I=\left(\delta_{i j}\right)_{n \times n}$ is the identity matrix. If trace $(A B) \leqslant C$ for some $C>0$, then we have

$$
|\operatorname{det} B| \leqslant(2 C)^{n} .
$$

Proof. We put $\bar{\mu}=$ the maximum eigenvalue of $B$. Then by nonnegative definiteness of $B$ we have $\bar{\mu} \leqslant\|B\|:=\left(\sum_{i, j=1}^{n} b_{i j}^{2}\right)^{1 / 2} \leqslant \sqrt{n} \bar{\mu}$. Hence we have

$$
\begin{aligned}
\bar{\mu} \leqslant \operatorname{trace}(B) & =\operatorname{trace}((I-A) B)+\operatorname{trace}(A B) \\
& \leqslant\|I-A\| \cdot\|B\|+\operatorname{trace}(A B) \\
& \leqslant\left[n^{2} \cdot\left(\frac{1}{2 n^{2}}\right)^{2}\right]^{1 / 2} \cdot \sqrt{n} \bar{\mu}+C=\frac{\bar{\mu}}{2 \sqrt{n}}+C .
\end{aligned}
$$


This implies that $\bar{\mu} \leqslant 2 C$. At last, by the assumption that $B$ is nonnegative definite, we have $0 \leqslant \operatorname{det} B \leqslant \bar{\mu}^{n} \leqslant(2 C)^{n}$. The proof is finished.

The following lemma is a slight extension of Jensen's lemma (see, for example, [6, Lemma A.3]).

Lemma A.2. Let $U \subset M$ be a convex domain of $M$ and let $h \in W^{1,2}(U) \cap C(U)$ satisfy $\Delta h \leqslant \lambda$ on $U$ for some constant $\lambda \in \mathbb{R}$. Assume that point $\hat{x} \in U$ is a uniquely local minimum point of function $h$ on $U$. Let $\left\{y^{j}\right\}_{1 \leqslant j \leqslant n}$ be a local geodesic coordinate system around $\hat{x}$. For any $p=\left(p^{1}, \cdots, p^{n}\right) \in \mathbb{R}^{n}$, we set

$$
h_{p}(x):=h(x)+\sum_{i, j=1}^{n} p^{j} y^{j}(x) .
$$

Then for any $r, \delta>0$ sufficiently small, (namely, they are smaller than a constant $\delta_{0}$ depending on the bounds of sectional curvature on $U$,) the set

$$
K=\left\{x \in B_{r}(\hat{x}): \exists p \in B_{\delta}(0) \text { for which } h_{p} \text { has a local minimum at } x\right\}
$$

has positive measure, where $B_{\delta}(0):=\left\{p \in \mathbb{R}^{n}:\|p\|<\delta, \forall 1 \leqslant j \leqslant n\right\}$.

Proof. Fixed any sufficiently small $r$, if $\delta$ is small enough, then for any $p \in B_{\delta}(0)$, there exists a local minimum of $h_{p}$ lying in the interior of $B_{r}(\hat{x})$, since $h$ has the unique minimum at $\hat{x}$. We will split the proof into two steps, as in the argument of [6, Lemma A.3].

(i) We assume for the moment that $h$ is of $C^{2}$ near $\hat{x}$. Let $\left(g_{i j}\right)_{n \times n}$ be the local Riemannian metric around $\hat{x}$ with respect to the coordinate system $\left\{y^{j}\right\}_{1 \leqslant j \leqslant n}$. There exists a number $r_{0}>0$, depending on the curvature on $U$, such that, for all $1 \leqslant i, j, k \leqslant n$,

$$
\left|g_{i j}(x)-\delta_{i j}\right| \leqslant \frac{1}{10 n^{2}}, \quad\left|\partial_{k} g_{i j}(x)\right| \leqslant 1, \quad \forall x \in B_{r_{0}}(\hat{x}) .
$$

Fixed any $r \in\left(0, r_{0}\right)$, it suffices to show that $K$ has positive measure with respect to the Euclidean measure on $\left(B_{r}(\hat{x}), \delta_{i j}\right)$.

Now we consider the elliptic operator

$$
L h:=\sum_{1 \leqslant i, j \leqslant n} \partial_{i}\left(a_{i j} \partial_{j} h\right) \quad \text { with } \quad a_{i j}=g^{i j} \sqrt{\operatorname{det}\left(g_{i j}\right)} .
$$

It is easily seen that there exists a constant $C(n)$ such that for all $x \in B_{r}(\hat{x})$ and for all $1 \leqslant i, j, k \leqslant$ $n$, they hold

$$
\left|a_{i j}(x)-\delta_{i j}\right| \leqslant \frac{1}{4 n^{2}}, \quad\left|\partial_{k} a_{i j}\right| \leqslant C(n), \quad L h \leqslant C(n) \cdot \lambda .
$$

Since $D h+p=D h_{p}=0$ holds for every minimum points of $h_{p}$, we have

$$
D h(K) \supseteq B_{\delta}(0) .
$$

Here $D h$ (and the following $D^{2} h$ ) is the (2-order) differential of $h$ with respect to the Euclidean metric on $B_{r}(\hat{x})$. Moreover, for every minimum points of $h_{p}$, we have that $D^{2} h_{p}=\left(\partial_{i} \partial_{j} h_{p}\right)$ is nonnegative definite and that

$$
\begin{aligned}
\sum_{1 \leqslant i, j \leqslant n} a_{i j} \partial_{i} \partial_{j} h_{p} & =\sum_{1 \leqslant i, j \leqslant n} \partial_{i}\left(a_{i j} \partial_{j} h_{p}\right) \quad\left(\text { since } \partial_{j} h_{p}=0\right) \\
& =L h+L\left(\sum_{k=1}^{n} p_{k} \cdot x_{k}\right)=L h+\sum_{1 \leqslant i, j \leqslant n} p_{j} \cdot \partial_{i} a_{i j} \\
& \leqslant C(n) \cdot \lambda+C(n) n^{2} \delta \leqslant C_{1}(n, \lambda),
\end{aligned}
$$


for a constant $C_{1}>0$. By using Lemma A.1 for $B=D^{2} h_{p}$, we have $\left|\operatorname{det} D^{2} h\right|=\left|\operatorname{det} D^{2} h_{p}\right| \leqslant$ $\left(2 C_{1}\right)^{n}$ for all $x \in K$. Thus,

$$
\mathcal{L}^{n}\left(B_{\delta}(0)\right) \leqslant \mathcal{L}^{n}(D h(K)) \leqslant \int_{K}\left|\operatorname{det} D^{2} h\right| d x \leqslant \mathcal{L}^{n}(K) \cdot\left(2 C_{1}\right)^{n},
$$

where $\mathcal{L}^{n}(K)$ is the Euclidean measure of $K$. This completes the proof for the smooth case.

(ii) In the general case, in which $h$ need not to be smooth, we will approximate it via heat flows. This is the reason that we have to assume that $U$ is convex.

Let $\left\{P_{t} h\right\}_{t \geqslant 0}$ be the heat flow with Neumann boundary value condition on $U$, with the initial data $P_{0} h=h$. It is clear that $P_{t} h$ is smooth for any $t>0$. By maximum principle, we have

$$
\Delta P_{t} h=P_{t} \Delta h \leqslant \lambda, \quad \forall t>0 .
$$

The corresponding set $K_{t}$ obey the above estimates in (i) for small $t>0$. In particular, the measure of $K_{t}$ is bounded from below by a constant $C(\delta, \lambda, n)>0$ uniformly on $t>0$.

At last, by using the convexity of the boundary of $U$ and that the curvature of $M$ is bounded on $U$, (in particular, the Ricci curvature on $U$ is bounded from below, the Li-Yau gradient estimates for solutions of the heat flow implies that $P_{t} h$ converge uniformly to $h$ on $B_{r}(\hat{x}) \subset \subset U$. Notice that $K \supset \liminf _{t \rightarrow 0} K_{t}$. The result now follows.

Now the perturbation Lemma 4.3 is a corollary as follows.

Corollary A.3. Let $U \subset M$ be a convex domain of $M$ and let $h \in W^{1,2}(U) \cap C(U)$ satisfy $\Delta h \leqslant \lambda$ on $U$ for some constant $\lambda \in \mathbb{R}$. Assume that point $\hat{x} \in U$ is one of minimum points of function $h$ on $U$. Assume a subset $A \subset U$ has full measure. Then for any $r, \delta>0$ sufficiently small, there exists a smooth function $\phi$ on a neighborhood of $\hat{x}, B_{r_{0}}(\hat{x})$, such that $h+\phi$ has a local minimum point in $B_{r}(\hat{x}) \cap A$ and that

$$
|\phi|+|\nabla \phi|+|\operatorname{Hess}(\phi)| \leqslant \delta, \quad \forall x \in B_{r_{0}}(\hat{x}) .
$$

Proof. Fix any $r, \delta$ sufficiently small. We put $h_{1}:=h+\delta|\hat{x} x|^{2} /(10 n)$. Then $h_{1}$ has a unique minimum at $\hat{x}$. Since $\Delta h_{1} \leqslant \Delta h+C\left(n, k_{0}\right) \cdot \delta$ by Laplacian comparison on $M$, the above Lemma implies that $h_{1}+\sum_{j=1}^{n} p^{j} y^{j}$ has a local minimum at a point in $B_{r}(\hat{x}) \cap A$ and that $0 \leqslant p^{j} \leqslant \delta / 2$, $1 \leqslant j \leqslant n$. Now, the function

$$
\phi:=\delta|\hat{x} x|^{2} /(10 n)+\sum_{j=1}^{n} p^{j} y^{j}
$$

defined on a coordinate neighborhood $B_{r_{0}}(\hat{x})$. Notice that $\mid$ Hess $y^{j} \mid \leqslant C\left(k_{0}\right)$ for some constant $C\left(k_{0}\right)$ depending on $k_{0}$, a bound of $\left|\sec _{M}\right|$ on $U$. This implies that

$$
|\phi|+|\nabla \phi|+|\operatorname{Hess}(\phi)| \leqslant \delta \cdot C\left(n, k_{0}\right), \quad \forall x \in B_{r_{0}}(\hat{x}) .
$$

The proof is finished.

\section{REFERENCES}

[1] D. Burago, Y. Burago \& S. Ivanov, A Course in Metric Geometry, Graduate Studies in Mathematics, vol. 33, AMS (2001).

[2] C. Breiner, A. Fraser, L.-H. Huang, C. Mese, P. Sargent \& Y. Zhang, Regularity of harmonic maps from polyhedra to CAT(1) spaces, preprint.

[3] J. Chen, On energy minimizing mappings between and into singular spaces, Duke Math. J. 79 (1995), 77-99.

[4] S. Y. Cheng, Liouville theorem for harmonic maps. Proc. Sympos. Pure Math. 36 (1980) 147-151.

[5] H. I. Choi, On the Liouville theorem for harmonic maps, Proc. Amer. Math. Soc. 85 (1982) 91-94.

[6] M. Crandall, H. Ishii \& P-L. Lions, User's guide to viscosity sulutions of second order partial differential equations, Bull. AMS, Vol. 27(1), 1-67, 1992. 
[7] G. Daskalopoulos, C. Mese, Harmonic maps from a simplicial complex and geometric rigidity, J. Differential Geom. 78 (2008), 269-293.

[8] G. Daskalopoulos, C. Mese, Fixed point and rigidity theorems for harmonic maps from simpticial complexes into NPC spaces, Geom. Dedica, 141 (2009), 33-57.

[9] G. Daskalopoulos, C. Mese, Harmonic maps between singular spaces I, Comm. Anal. Geom. 18 (2010), 257337.

[10] G. Daskalopoulos, C. Mese \& A. Vdovila, Superrigidity of hyperbolic buildings, Geom. Funct. Anal. 21 (2011), no. 4, 905-919.

[11] J. Eells, B. Fuglede, Harmonic maps between Riemannian polyhedra, Cambridge Tracts Maths., 142, Cambridge University Press, Cambridge (2001).

[12] B. Freidin, A Bochner Formula for Harmonic Maps into Non-Positively Curved Metric Spaces, available at arXiv:1605.08461 2 .

[13] B. Freidin, Y. Zhang, A Liouville-type theorem and Bochner formula for harmonic maps into metric spaces, available at arXiv: $1805.04192 \mathrm{v} 1$.

[14] B. Fuglede, Hölder continuity of harmonic maps from Riemannian polyhedra to spaces of upper bounded curvature, Calc. Var. PDE 16(4) (2003), 375-403.

[15] B. Fuglede, Harmonic maps from Riemannian polyhedra to geodesic spaces with curvature bounded from above, Calc. Var. PDE 31(1) (2008), 99-136.

[16] M. Giaquinta, E. Giusti, On the regularity of the minima of variational integrals, Acta Math., 148 (1982) 31-46.

[17] M. Giaquinta, E. Giusti, The singular set of the minima of certain quadratic functionals. Ann. Scuola Norm. Sup. Pisa, Cl. Sci., (4) (1984) 11, 45-ÅC55.

[18] G. Gregori, Sobolev spaces and harmonic maps between singular spaces, Calc. Var. PDE 7 (1998), 1-18.

[19] M. Gromov, R. Schoen, Harmonic maps into singular spaces and p-adic superrigidity for lattices in groups of rank one, Publ. Math. IHES 76 (1992), 165-246.

[20] C.-Y. Guo, Harmonic mappings between singular metric spaces, available at https://arxiv.org/abs/1702.05086.

[21] J. Heinonen, P. Koskela, N. Shanmugalingam \& J. Tyson, Sobolev classes of Banach space-valued functions and quasiconformal mappings, J. Anal. Math. 85 (2001), 87-139.

[22] S. Hildebrandt, H. Kaul \& K.-O. Widman, An existence theorem for harmonic mappings of Riemannian manifolds, Acta Math., 138 (1977), no.1-2, 1-16.

[23] B. Hua, S. Liu \& C. Xia, Liouville theorems for f-harmonic maps into Hadamard spaces, Pacific J. Math., 290 (2017), no.2, 381-402.

[24] W. Ishizuka, C. Y. Wang, Harmonic maps from manifolds of $L^{\infty}$-Riemannian metrics, Calc. Var. PDE, 32 (2008), 287-405.

[25] W. Jäger, H. Kaul, Rotationally symmetric harmonic maps from a ball into a sphere and the regularity problem for weak solutions of elliptic systems, J. Reine. Angew. Math., 343 (1983), 146-161.

[26] J. Jost, Equilibrium maps between metric spaces, Calc. Car. PDE 2 (1994), 173-204.

[27] J. Jost, Generalized harmonic maps between metric spaces, Geometric Analysis and the Calculus of Variations for Stefan Hildebrandt, J. Jost (editor), 143-174, Intern. Press, (1996).

[28] J. Jost, Generalized Dirichlet forms and harmonic maps, Calc. Var. PDE 5, (1997), 1-19.

[29] J. Jost, Nonlinear Dirichlet forms, New directions in Dirichlet forms, J. Jost and W. Kendall and U. Mosco and M. Röckner and K.Th. Sturm (editor), 1-47, International Press/AMS, (1998).

[30] B. Kirchheim, Rectifiable metric spaces: local structure and regularity of the Hausdorff measure, Proc. Amer. Math. Soc. 121 (1994), no. 1, 113-123.

[31] N. Korevaar, R. Schoen, Sobolev spaces and harmonic maps for metric space targets, Comm. Anal. Geom. 1 (1993), 561-659.

[32] K. Kuwae, T. Shioya, Sobolev and Dirichlet spaces over maps between metric spaces, J. Reine Angew. Math., 555, 39-75, (2003).

[33] F. H. Lin, Analysis on singular spaces, Collection of papers on geometry, analysis and mathematical physics, 114-126, World Sci. Publ., River Edge, NJ, (1997).

[34] F. H. Lin, C. Y. Wang The analysis of harmonic maps and their heat flows, World Sci. Publ., River Edge, NJ, (2008), ISBN: 978-981-277-952-6.

[35] C. Mese, The curvature of minimal surfaces in singular spaces , Comm. Anal. Geom., 9(1) (2001), 3-34.

[36] C. Mese, Harmonic maps into spaces with an upper curvature bound in the sense of Alexandrov, Math. Z., 242 (2002), 633-661.

[37] C. B. Morrey, The problem of Plateau on an Riemannian manifold, Ann. Math., 49. 807-851.

[38] S.-I. Ohta, Cheeger type Sobolev spaces for metric space targets. Potential Anal. 20 (2004), no. 2, 149-175. 
[39] R. Schoen, S. T. Yau, Harmonic maps and topology of stable hypersurfaces and manifolds of nonnegative Ricci curvature, Comm. Math. Helv., 39 (1976), 333-341.

[40] R. Schoen, S. T. Yau, Lectures on differential geometry, International Press, Boston, 1994.

[41] R. Schoen, K. Uhlenbeck, A regularity theory for harmonic maps, J. Differ. Geom. 17, 307-335 (1982).

[42] R. Schoen, K. Uhlenbeck, Regularity of minimizing harmonic maps into the sphere, Invent. math., 78, 89-100 (1984).

[43] T. Serbinowski, Harmonic Maps into Metric Spaces with Curvature Bounded from above, Ph. D. Thesis: University of Utah (1995).

[44] Z. Sinaei, Riemannian Polyhedra and Liouville-Type Theorems for Harmonic Maps, Anal. Geom. Metr. Spaces (2014) 2, 294-318.

[45] K-T. Sturm, A semigroup approach to harmonic maps, Potent. Anal. 23 (2005) 225-277.

[46] H. C. Zhang, X. P. Zhu, Local Li-Yau's estimates on $R C D^{*}(K, N)$ metric measure spaces, Calc. Var. (2016) 55:93.

[47] H. C. Zhang, X. P. Zhu, Lipschitz contunuity of harmonic maps between Alexandrov spaces, Invent. math. (2018) 211:863-934.

Department of Mathematics, Sun Yat-sen University, Guangzhou 510275,

E-MAIL ADDRESS: ZHANGHC3@MAIL.SYSU.EDU.cN

Department of Mathematics and Statistics, University of Helsinki, FI-00014 University of Helsinki,

E-MAIL ADDRESS: XIAO.X.ZHONG@HELSINKI.FI

Department of Mathematics, Sun Yat-sen University, Guangzhou 510275,

E-MAIL ADDRESS: STSZXP@MAIL.SYSU.EDU.CN 